\title{
Delivery Actuator for a Transcervical Sterilization Device
}

M. Rehan ${ }^{1}$, J. Coleman ${ }^{2}$ and A. G. Olabi ${ }^{{ }^{*}}$

${ }^{1}$ School of Mechanical and Manufacturing Engineering, Dublin City University, Glasnevin, Dublin 9, Ireland

${ }^{2}$ Alta Science, Trinitas House, 2012 Orchard Avenue, Citywest Campus, Citywest, Dublin 24, Ireland

*Corresponding Author

A. G. Olabi

School of Mechanical and Manufacturing Engineering, Dublin City University, Glasnevin, Dublin 9, Ireland

Tel.: +3537007718

Fax: +353 7007148

E-mail address: abdul.olabi@dcu.ie

\section{Word Count}

Abstract: 291 words

Keywords: 6 words

Introduction: 581 words

Material and Methods: 4259 words

Result and Discussion: 788 words

Conclusion: 208 


\begin{abstract}
The use of delivery systems in the human body for positioning and deploying implants, such as closure devices, dilation balloons, stents, coils and sterilization devices, are gaining more importance to preclude surgical incisions and general anesthesia. The majorities of the nonsurgical medical devices are delivered in a low profile into human body form and subsequently require specialized operations for their deployment and release. An analogous procedure for permanent female sterilization is the transcervical approach that does not require either general anesthesia or surgical incision and uses a normal body passage. The objective of this paper is to detail the design, development and verification of an ergonomic actuator for a medical application. In particular, this actuator is designed for the deployment and release of an implant to achieve instant permanent female sterilization via the transcervical approach. This implant is deployed under hysteroscopic visualization and requires a sequence of rotary and linear operations for its deployment and release. More specifically, this manually operated actuator is a hand held device designed to transmit the required forces in a particular sequence to effect both implant deployment and release at a target location. In order to design the actuator and to investigate its mechanical behavior, a three-dimensional (3D) Computer Aided Design (CAD) model was developed and Finite Element Method (FEM) was used for simulations and optimization. Actuator validation was performed following a number of successful bench-top inair deployments and in-vitro deployments in animal tissue and explanted human uteri. During these deployments it was observed that the actuator applied the required forces to the implant resulting in successful deployment. Initial results suggest that this actuator can be used single handedly during the deployment phase. The ongoing enhancement of this actuator is moving towards “first-in- man" clinical trials.
\end{abstract}


Key words: Transcervical sterilization, actuator, Finite Element Method, Optimization, 3D modeling, Female sterilization. 


\section{Introduction}

Worldwide, female sterilization is the most widely used method of permanent contraception. The transcervical approach is an alternative to incisional procedures for interval tubal sterilization eliminating surgery and general anesthesia. However, development of a safe and effective transcervical method of sterilization is a challenging task [1], as it requires both reliable method of tubal occlusion and actuator for its safe delivery and effective deployment.

Numerous destructive and mechanical occlusive methods of transcervical sterilization have been studied, over the last 150 years [2]. The destructive methods mainly focused on chemical causticts, tissue adhesives, thermic induction and the Nd:YAG laser [3-4]. The majority of mechanical occlusion devices are applied hysteroscopically and occlusion can be achieved either by placing a pre-formed plug or device in to the uterotubal orifice or by formed-in-situ methods. Whether it is a preformed plug or a formed-in-situ method, the hysteroscopic sterilization method needs a special delivery device or an actuator for accurate placement, deployment and release of the occlusive system.

Following the technological advancements in optics and catheters, some new technologies based on mechanical methods such as the Adiana Complete Transcervical Sterilization System (Adiana, Redwood City, CA) and Essure system (Conceptus, Inc., San Carlos, CA) have evolved [2]. The Adiana procedure consists of applying radiofrequency energy to the fallopian tubes to ablate the epithelial lining and depositing a synthetic matrix in the lumen of the fallopian tube. The synthetic matrix is deployed hysteroscopically using a delivery catheter and an actuator [56]. The second mechanical method of hysteroscopic sterilization is the Essure ${ }^{\circledR}$ System (Conceptus) whose insert is a dynamically expanding micro-coil that is delivered into the 
proximal section of the fallopian tube using a guide wire and release catheter. The entire system is attached to an actuator handle, aligned for single-handed release of the device [7-8].

The previous work of the author involves the designing of the specialized magnetostrictive (MS) actuator for automotive applications [9-10] while this paper focuses on an entirely different mechanical actuator for medical applications. Most delivery actuators used in sterilization devices are mechanical and manual operated. In addition, these devices need to be ergonomically designed, cost effective, easy to use and disposable. Therefore, the innovative manual mechanical actuator detailed in this paper was designed in view of above mentioned characteristics. The primary objective of this actuator is the deployment and release of an implant [11] for occlusion of fallopian tubes in order to provide immediate and permanent transcervical female sterilization as shown in Fig. 1. The implant is miniature in size and requires precise movements for deployment and release. Therefore, designing a hand-held ergonomic actuator capable of applying all required forces within a minute and precise design space was a challenging task. To design and simulate the mechanical behavior of the actuator Finite Element Analysis (FEA) software ANSYS Workbench (WB) was used. The parametric model generated in Pro/E was integrated with ANSYS WB for FEA simulations and analysis/optimization [12, 13, manuscript submitted to Advances in Engineering Software]. The actuator was validated a number of times by successful deployments on the bench $(n>50)$, in animal tissue $(n=10)$ and in explanted human uteri ( $n=7)$. During deployments in the latter, it was observed that the actuator deployed the implant successfully and provided both instant and effective occlusion of the fallopian tube. Initial results suggest that this actuator can be used single handedly during deployment phase. It is anticipated that this actuator could be adapted to use for other medical devices and applications, which require manual actuations are required. 


\section{Material and Methods}

To comprehend the actuator design, an appreciation of the functionality of the implant is required. The implant, with a length of $6.5 \mathrm{~mm}$, an outer diameter $(\varnothing)$ of $1.535 \mathrm{~mm}$ and a thickness of $0.1 \mathrm{~mm}$, is made of annealed SS-316LVM. It features two sets of six slots at the distal and proximal segments. Post deployment, these slots determine the implant's final shape by formation of two sets of six wings. The implant includes an inner release tube designed to break after implant deployment. Fig. 2 depicts the comparison of the un-deployed and deployed implant.

The implant is designed to deploy and release in a five steps sequence:

Step 1: A counter-clockwise (CCW) torque of $15.4 \mathrm{~N}$-mm, which generates an out-of-plane displacement in the implant's distal slots.

Step 2: An axial compression of $25 \mathrm{~N}$ that plastically deforms the displaced slots into the shape of the first set of six distal wings.

Step 3: A clockwise (CW) torque of $16 \mathrm{~N}$-mm, which generates an out-of-plane displacement in the implant's proximal slots

Step 4: An axial compression of $25 \mathrm{~N}$ that plastically deforms the displaced slots into the shape of second set of six proximal wings.

Step 5: A tensile force of $70 \mathrm{~N}$ on the release tube breaks the link of the implant to the actuator.

The implant is inserted hysteroscpically in low profile form, by means of a flexible guide tube and inner guide wire. As the implant requires torque and compression for deployment, both the guide tube and wire serves the purpose of torque transmission and compression to the implant. Because of their flexibility, the guide tube and guide wire lacks one-to-one force transfer property. In addition, due to their construction, they inherit dissimilar CW and CCW 
torqabilities. It was experimentally measured that to transfer a CCW torque of $15.4 \mathrm{~N}$-mm through a multifilament flexible cable (1x7), with a length 360mm and a Ø 0.7, a CCW rotation of $410^{\circ}$ is required. Alternately, to apply the same amount of $\mathrm{CW}$ torque requires a CW rotation of $530^{\circ}$ is required. Therefore, to transfer the above-mentioned forces to the implant, large rotational and translational displacements were required from the actuator.

The challenging task was to design an actuator that could provide controlled rotations of more than $360^{\circ}$. Fig. 3 shows an initial design of the actuator that was capable of providing the required displacements in a specified sequence. In order to apply a CCW torque of $15.4 \mathrm{~N}$-mm and $\mathrm{CW}$ torque of $16 \mathrm{~N}-\mathrm{mm}$, a rotations of $410^{\circ}$ and $970^{\circ}$ were required respectively. To achieve these rotation angles, three telescopic cylinders were used in cascade. These cylinders were slidably and rotatably connected to each other through a pin-in-slot mechanism. A combination of slots and engagement of the pins in the cylinders provided the required rotations. The axial displacements were achieved through the helical shape of slots ends. As a result, the actuator was capable to provide the required displacements to the implant for deployment. Successful deployments of the implant on bench-top testing validated the operational concepts. During these testing and implant deployments, limitations were observed in the actuator design.

1. Actuator was designed to provide fixed amount of rotations and linear displacements, it could only be used with the specific guide wire for which it was designed.

2. There was no mechanism to prevent any inadvertent reverse rotations and to avoid backlash (opposing torque) from the implant, guide wire and guide tube.

3. The actuator release lever interfered with the hysteroscope during placement of the implant. 
All the above-mentioned limitations lead to the necessity of an improved actuator design presented in this paper.

\section{Actuator Design}

The ergonomically designed actuator presented in this paper controls the deployment and release of the implant at the target location by applying the required forces in a specified sequence. The designing and 3D modeling of the actuator was performed using CAD software Pro/Engineer Wildfire 3.0 (Pro/E) is shown in Fig. 4.

\section{Basic Configuration:}

The actuator comprises a handle-body, with a diameter of $25 \mathrm{~mm}$, which is adapted to hold the actuator. At the distal end, a fore-body, with maximum Ø $32 \mathrm{~mm}$ is slidably and rotatably connected to the handle-body. These optimal diameters of the handle-body and fore-body were adapted from the previous work of Yong et. al. [14], [15]. The addition of a compression spring in between the fore-body and the handle-body automates the compression steps of the implant deployment sequence. This reduces the deployment and release sequence from five steps as mentioned earlier to three steps. In order to acquire a $25 \mathrm{~N}$ compressive force to deploy both wings, a compression spring with spring rate of $1.67 \mathrm{~N} / \mathrm{mm}$ was used. At the initial stage, this spring is deflected $18 \mathrm{~mm}$ thereby applying a force of $30 \mathrm{~N}$, which reduces to $25 \mathrm{~N}$ for the proximal wings.

This actuator topology was based on a ratchet wheel and pawl pin configuration. The fore-body is therefore operatively connected to handle-body through a ratchet mechanism in which the handle-body incorporates ratchet wheels and the fore handle includes a pawl pin as shown in Fig.

4. The ratchets were incorporated into the actuator to control the precise CW and CCW rotations by way of restricting any inadvertent reverse rotations. In addition, they prevent backlash from 
the implant and guide system during torque application. The precision of the latching was dependent on the number of teeth in the ratchet wheel. The design of the size of the ratchet wheel, number of teeth and size of pawl pin was based on the preferred size of actuator, the minimum feature size of machining, strength of the teeth and positive locking of teeth and pawl pin. The actuator also features a safety pin, which locks the actuator, preventing accidental deployment during handling or transportation. This safety pin needs to be removed prior to the deployment sequence.

The proximal end of the handle-body includes a release mechanism, which controls the release of the implant. This release mechanism includes an externally threaded release shaft and an internally threaded release knob. The release shaft is slidably connected to the handle-body and the release knob is rotatably connected to the handle-body. The power screw mechanism in between the release knob and release shaft converts the rotary motion of the release knob into axial linear motion of the release shaft. A tensile force of $70 \mathrm{~N}$ is required to release the implant. The power screw mechanism provides this force by converting the torque applied on the release knob. The actuator was designed to limit this release torque to $520 \mathrm{~N}$-mm i.e. that a human hand can apply with an index finger on a cylinder of Ø 25 mm [15]. ISO (International Organization for Standards) basic threads were selected for this mechanism because of the available machining resources. However, in this mechanism the release torque can be reduced by using square threads. Considering the size of the actuator, M10 coarse threads (pitch $1.5 \mathrm{~mm}$ ) were preferred. A standard ISO M10 thread profile with basic dimensions is shown in Fig. 5 [16]. The torque required to apply the force of $70 \mathrm{~N}$ was calculated using a force balance diagram of a standard ISO threads with a single start as shown in Fig. 6. 
From Fig. 6, $L=n p$ for a multi start drive, $n$ being the number of start threads. Here $L$ is the half pitch, $N$ is the normal reaction and $\mu N$ is the frictional force.

For equilibrium

$P-\mu N \cos \alpha-N \sin \alpha=0$

$F+\mu N \sin -N \cos \alpha=0$

Torque transmitted to apply load is given by

$T_{R}=F \frac{d m}{2}$

Rearrangement of Eq. (1) results in

$T_{R}=F \frac{d m(\mu \cos \alpha+\mu \sin \alpha)}{2(\cos \alpha-\mu \sin \alpha)}$

Since,

$\tan \alpha=\frac{L}{\pi d m}$

$T_{R}=F \frac{d m(\mu \pi d m+L)}{2(\pi d m-\mu L)}$

Were $\alpha=30^{\circ}, d m=9.026, L=0.75, F=70 \mathrm{~N}$ and $\mu=0.74$ for steel on steel

This analysis suggests that a torque of $242.13 \mathrm{~N}-\mathrm{mm}$ is required on the release knob to attain a tensile force of $70 \mathrm{~N}$ from the release mechanism resulting in release of the implant from the actuator.

\section{Mode of Operation:}

The three step operational sequence of the actuator shown in Fig. 7(a-d) is detailed below:

a) During the preliminary stage, the safety pin is engaged and the actuator is fully locked, preventing the implant from accidental deployment, handling loads and spring compressive force. The pawl pin is in the first (distal) ratchet, the compression spring is $18 \mathrm{~mm}$ deflected and exerting a $30 \mathrm{~N}$ force absorbed by safety pin. 
b) In the first step, the safety pin is removed, shifting the $30 \mathrm{~N}$ of compressive force from the safety pin to the implant. The fore-body can now be rotated CCW and the pawl pin latches into the ratchet wheel (for distal wings). This operation applies torque on the implant, allowing the distal slots to displace outward. When the compressive force overcomes the internal resistance of the implant slots, the distal wings are formed by the compression of the slots. During this process, the pawl pin is pushed in the proximal ratchet locking the CCW rotation. At the end of this step, the spring deflection is reduced from $18 \mathrm{~mm}$ to $15.5 \mathrm{~mm}$ thereby reducing the spring force to $25 \mathrm{~N}$.

c) In the second step, the fore-body can only be rotated CW and the pawl pin latches in the ratchet wheel (for proximal wings). The compression spring is $15.5 \mathrm{~mm}$ deflected and exerting a force of $25 \mathrm{~N}$ to the implant. During the process of wings formation, the pawl pin is driven into the release wheel by the compression spring. At the end of this step, the pawl pin is settled in the release wheel, which locks all rotations and backward translation of the fore-body. Spring deflection is further reduced from $15.5 \mathrm{~mm}$ to 13 $\mathrm{mm}$, thus reducing the spring force to $21 \mathrm{~N}$.

d) In the final step, the only possible operation is the rotation of the release knob. The release knob can be rotated CW to effect the release shaft which moves axially backward. This axial movement of the release shaft away from the implant exerts a tensile force on the implant's release mechanism. At a release torque of $242.13 \mathrm{~N}-\mathrm{mm}$, the corresponding tensile force reaches a value of $70 \mathrm{~N}$, causing the release mechanism to break, thus releasing the implant from the delivery actuator. 


\section{FEA and Optimization}

Designing a device through trial and error based prototyping and experimentation is both an expensive and time-consuming process. Therefore, FEA simulation in conjunction with experimental testing was used to achieve the optimum design and first prototype of the device. Finite Element Analysis (FEA) software ANSYS Workbench (WB) was used in integrated mode with Pro/E for FEA simulations and optimization. These simulations were performed to simulate functionality and to investigate the mechanical behavior of the actuator under deployment, release and handling forces as mentioned in Table I.

Design optimization of the actuator was performed using Design Xplorer, a module of ANSYS WB. The idea behind using DesignXplorer was to use its powerful capabilities of importing a model from Pro/E and implementing a chosen design change back into the original model [1112]. DesignXplorer uses statistical methods for optimization thus allowing the designer to reach the optimized design in a very short time thereby reducing the design cycle time and increasing the productivity of both analyst and designer. The objective of these optimization studies was to investigate the mechanical behavior of the actuator and to minimize its mass. All the actuator components were analyzed and optimized individually in this process. FEA analysis was defined according to the actual scenario in the simulation module of ANSYS WB. A biocompatible grade of stainless steel SS-316, a nickel-chromium-molybdenum alloy steel, was chosen for the actuator. The material properties of SS-316 were assigned in the ANSYS WB environment. Young's modulus $E$ used for this material was $193 \mathrm{GPa}$, Poisson ratio $v$ was 0.3 , yield strength $\sigma_{y}$ was $690 \mathrm{MPa}$ and the ultimate tensile strength $\sigma_{u}$ was $860 \mathrm{MPa}$. A deterministic approach based on Design of Experiments (DOE) was used to study the response of design variables. During the optimization study of each component, the design variables were defined according to the 
respective geometry and boundary conditions. The upper and lower limiting values of design variables were assigned to parameters leading to the generation of Automatic Design Points (ADP). Based on the ADP, the response of design variables with respect to maximum von-Mises stress was plotted and reviewed to the best feasible design point. Goal driven optimization was performed based on ADP study and a candidate design based on current goals was generated with the best candidate design being selected and inserted as a soft design. The best candidate design was used to generate the hard reference design by performing FEA analysis in ANSYS WB environment. Finally, the reference design point was rated and the current (optimum) parameter values for the best design were automatically updated from the DesignXplorer into the parameter manager consequently updating Pro/E model. A final analysis was performed in the WB environment to verify the results obtained from the design optimization.

The actuator was designed and optimized to withstand the deployment and release forces. The mechanical loads were evaluated with the worst-case scenarios of the dominant handling loads. All the actuator components were optimized against their respective loadings as mentioned in Table I. The objective of these optimization studies was to minimize the mass of the actuator components. Therefore, the minimum volume was defined as objective variable (goal), the maximum von-Mises stress with the limiting values of $680 \pm 10 \mathrm{MPa}$ was defined as the state variable (constraint). The design optimization studies of individual components are discussed below:

\section{Fore-body:}

The fore-body was optimized against the deployment and release loads. The fore-body is physically attached to the guide tube by a socket set screw. Thus, torque and compression is transmitted to the guide tube through the socket set screw hole. In order to simulate the fore- 
body separately, the fixed displacement boundary condition was applied on the screw hole. Fig. 8(a) shows all the applied boundary conditions for the optimization study. The proximal end of the fore-body is constrained radially in the handle-body. Therefore, the cylindrical boundary condition was applied on this end. A moment of $16 \mathrm{~N}$-mm was applied on the grip surface having a maximum diameter and a compressive force of $70 \mathrm{~N}$ was applied on the rear surface. In the optimization study, the design parameters insrt_end_thk, cone_thk, rear_cyl_thk and mid_srf_wdth were defined for the fore-body thicknesses of the insert end, conical end, rear cylinder and the middle surface. The volume of the fore-body was meshed with hexahedron elements as shown in Fig. 8(b). An optimization study was performed to achieve an optimum volume while satisfying the constraints on the maximum von-Mises stress. The final analysis was performed on the optimum geometry to verify the optimization results. The von-mises stress distribution and the total deformation in the optimized model are shown in Fig. 8(c) and 8(d) respectively.

\section{Handle-body:}

The handle-body is designed to be gripped by the hand during usage of the actuator. Therefore, it was analyzed against both the maximum grip force of a human hand and the forces required for implant deployment and release as mentioned in Table I. The handle-body retains the fore-body inside the distal portion, the ratchet shaft at the mid portion and the release knob at the proximal end. The boundary conditions were applied to represent this whole scenario as shown in Fig. 9(a). A human hand wearing a latex glove can apply a grip force of $826 \mathrm{~N}$ on a $\varnothing 25 \mathrm{~mm}$ cylindrical body [17]. Hence, a force of $826 \mathrm{~N}$ in the form of pressure was applied on the outer surface of the handle-body. The release knob exerts a $70 \mathrm{~N}$ axial force during implant release, which was applied at the proximal end of the handle-body. The objective and state function of 
the optimization study were the same as detailed earlier. The design parameters frnt_cyl_thk and rear_cyl_thk were defined for the front and rear segment thicknesses of the handle-body. The parametric model was meshed with hexahedron elements as shown in Fig. 9(b). Optimization was performed on the initial design and the final analysis was performed on the optimum geometry to verify the optimization results. Fig. 9(c) and 9(d) shows the von-Mises stress distribution and total deformation in the final validation analysis.

\section{Ratchet Mechanism:}

During deployment, the only force experienced by the ratchet mechanism is the frictional force between the pawl pin and the ratchet wheel. The worst-case scenario for the ratchet mechanism would be an inadvertent reverse torque produced by mishandling of the actuator. The torque a human hand can apply on a Ø 25mm cylindrical body with an index finger and thumb is $520 \mathrm{~N}$ mm [14]. This value was also experimentally validated by testing the actuator using a torque meter. The ratchet mechanism was analyzed against this torque value. Boundary conditions were applied to simulate the actual conditions as shown in Fig. 10(a) in which fixed displacement were applied on the surfaces of the pawl pin, in contact with the fore-body. A moment of $520 \mathrm{~N}$-mm was applied on the inner cylindrical surface ratchet wheel. Surface-tosurface frictional contact was defined between the pawl pin and ratchet wheel with a frictional coefficient of 0.74. Fig. 10(b) shows the model meshed with hexahedron elements. The design parameters were defined for both the ratchet wheel and the pawl pin. The parameters teeth_ang, teeth_width,pin_up_dia and pin_lwr_dia were defined for the teeth angle, teeth width, pin upper diameter and pin lower diameter. An optimization study was performed after defining the objective and state function. Fig. 10(c) and 10(d) shows the von-Mises stress distribution and the total deformation in the ratchet wheel and pawl pin. 


\section{Release Mechanism:}

Release forces were applied on both the release knob and the shaft to investigate their mechanical behavior. In order to analyse the release mechanism, surface-to-surface frictional contact was defined in between the release knob and release shaft. To simulate the actual conditions those surfaces of the release knob, which remains in contact with the handle-body were constrained along the longitudinal axis. Fig. 11(a) details the boundary conditions applied on the assembly model. The mathematically calculated release torque of $242 \mathrm{~N}$-mm was applied on the outer surface of the release knob. A tensile force of $70 \mathrm{~N}$ was applied on the release shaft along the longitudinal axis. As Fig. 11(b) shows the model was meshed with tetrahedron elements and static analysis was performed to evaluate the stresses. The total deformation and the von-Mises stress distribution in the release knob and the release shaft are shown in Fig. 11(c) and 11(d) respectively.

\section{Development and testing}

\section{Prototyping:}

A stainless steel prototype was fabricated using a Computer Numerical Control (CNC) lathe for high accuracy requirement. Ratchet wheels were fabricated using Wire Cutting Machine. All engineering drawings were developed using Pro/E software and CNC programs were directly generated using Pro/NC a module of Pro/E. Fabrication was done by 140-CNC lathe, Schaublin Machines SA. All machined critical dimensions were inspected using Video Inspection Probe, Deltronic Inc. and were found within a tolerance $\pm 0.05 \mathrm{~mm}$. The manufactured prototype shown in Fig. 12(a) was used for various mechanical testing and validation of the actuator mechanisms. In addition, it was used for functionality test by deploying and releasing the implant on the testbench. Fig. 12(b) details the exploded view of the actuator. 


\section{Torque Testing on Handle-body:}

The torque a human hand can apply on a Ø $25 \mathrm{~mm}$ cylindrical body with an index finger and thumb is $520 \mathrm{~N}-\mathrm{mm}$ [14]. This value used in FEA simulations was experimentally validated by measuring torque on the actuator using torque-measuring equipment. The torque measuring setup is shown in Fig. 13 (a) in which torque is being applied on the handle-body. The torque meter was held in a vise. Due to the limitation of the torque meter's chuck $\varnothing$, a solid shaft was used to interface the actuator with the torque meter. One end of this shaft was griped in the torque meter and the opposite insert shaped end into the fore-body. Once the task was setup, the torque meter was reset and the recording mode was set to peak torque value. The handle-body was held with an index finger and thumb as per in normal operation mode. The torque was applied on the handle-body in both CW and CCW directions and peak torque values were documented. This recorded torque was compared with the available values from the previous studies [14], [15].

\section{Torque Testing on Release Knob:}

The torque on the release knob was measured by breaking the release tube inside the actuator. The setup to measure the torque to release the implant by breaking the release tube was quite specific. The release tube, with a length of $7 \mathrm{~mm}$, an outer $\varnothing$ of $1 \mathrm{~mm}$ and a thickness of 0.125 mm is made of hardened SS-316LVM tube and includes a pair of slots. This symmetric pair of laser cut slots forms an arc shape at both ends and a rectangular pattern in between. The gap in between this pair of slots forms a neck region, which is designed to break at a specified load. In order to hold the release tube inside the actuator, two lengths of SS-316 wires were laser welded at both ends of release tube. The release tube was positioned at the center of the actuator and the end wires were clamped as shown in Fig 13(c). One end of the wire was griped at the distal end of the fore-body using "guide tube insert set screw (Fig. 4)" and other end in the release shaft 
using "guide wire lock screw (Fig. 4)”. On completion of setup the torque measuring equipment was set to peak mode and torque was applied on the release knob as shown in Fig. 13 (b). Because of the power screw mechanism the applied torque on the release knob acted as a tensile force on the release tube. This torque was applied until breakage of the release tube. This measured torque was compared with the theoretically calculated torque value.

\section{Compression Testing:}

The compression force applied by the actuator for the formation of the wings was validated using a tensile testing machine (Lloyd Inc.). Here the handle-body was clamped in the upper jaw of the tensile testing machine and the fore-body was constrained axially in the lower jaw as shown in Fig. 14. The machine was programmed from the console to simulate the sequence of compression forces applied by the actuator. The three step sequence includes, firstly the compression force exerted by the actuator on the implant at initial stage; secondly, the compression force at the end of distal wings deployment; thirdly, the compression force at the end of proximal wings deployment. This sequence was executed and the compression forces at the end of each step were compared with the designed values.

The compression forces during the formation of the wings were also measured. For this measurement, the same setup mentioned above was used except an implant was loaded on the actuator using a smaller guide tube. The implant was deployed in this setup and the compression forces were measured through the load cell of the machine. The compression force achieved during this study was compared with the values of simulated values. 


\section{Functionality Testing}

Bench Top: The actuator was evaluated numerous times $(n>50)$ in the laboratory. The evaluations included deploying implant using actuator in air and in-vitro on the test bench. The bench-top in air and in-vitro actuator testing was one of the main sources for design verification and follow up developmental work. In-vitro bench testing $(\mathrm{n}=10)$ was carried out on both porcine tissue and fallopian tubes. These tests were performed to validate the functionality of the actuator against the external loads, i.e. the loading exerted by tissue on the implant. Bench-top air deployments of the implants were carried out to validate the functionality and mechanical behaviour of the actuator. Fig. 15 shows a bench top in air functionality test of the actuator. The actuator provided precisely the entire rotary and translatory motions in a particular sequence for implant deployment and release. The actuator usage for implant deployments was also performed under a microscope to both visualize and examine the wing formation and shape. The shape of the implant deployed by actuator was compared with the results of the implant FEA simulations [11] to validate the effectiveness of the actuator. To evaluate the accuracy of the actuator, the values of rotary displacement about the longitudinal axis and translatory displacements along the same axis, required for deployment of implant, were measured during the deployment process using video inspection probe.

Explanted Uteri studies: In-vitro deployments of the implant into explanted human uteri were performed using actuator as shown in Fig. 16. These studies $(n=7)$ were performed to validate the functionality, deliverability and accuracy of the actuator during clinical usage. The actuator deployed the implant bilaterally into the tubal ostia through the 5-French $(1.67 \mathrm{~mm}$ internal diameter) operating channel of a standard hysteroscope. Following deployment, the implant was released from the actuator using the actuator release mechanism. The procedure was repeated on 
the contra-lateral fallopian tube. Following successful implant deployment in both tubes, hydraulic pressure test of the uterus was performed to validate the occlusion of fallopian tubes [11]. Finally, the ostium and tubes were dissected to examine the placement and deployment of the implant in the intramural section of the ostium. The implant along with some tissue was extracted to further examine the wing shape and the deployed implant. The implant deployed using actuator was compared with the theoretical designed profile.

\section{Results and Discussion}

The objective of this paper was to detail the design, development and testing of an actuator that can both safely and effectively deploy and release a permanent implant from a device used to perform permanent transcervical sterilization. The design of the actuator was accomplished through 3D modeling, FEA simulations, prototyping and testing (both mechanical and functional). The 3D model of the actuator generated in Pro/E was integrated with ANSYS WB for FEA simulations and optimization. FEA simulations were performed to investigate the mechanical behavior and functionality of the actuator against deployment, release and handling loads. Mesh convergence was performed on all the FE models of the actuator components. The effect of mesh density on the model accuracy was investigated using six different FE meshes detailed in Table II. The mesh type A is the coarsest and type F was the finest. The FE meshes were varied by varying the element size and the mesh relevance. To estimate the mesh convergence, the von-Mises stresses in each model were compared for each mesh refinement, keeping constant, the rest of the variables. Fig. 17(a-d) shows the mesh convergence of the actuator components to assess the necessary level of mesh refinement. Optimization was performed to achieve the minimum mass of the actuator. During the actuator components 
optimization studies, it was observed that the machining constraint was a limiting factor on design variables. It was observed that, even at thicknesses comparable to the implant, the vonMises stresses among the components were well below the maximum limiting values. Therefore, the optimum design was maintained based on the machining constraints. The response of various design variables versus von-Mises stress distribution is shown in Fig. 18(a-d).

The actuator in this study was fabricated using CNC lathe, milling machine and wire cutting machine. The CNC programs for the machines were generated using Pro/NC. Fabricated actuator was inspected on coordinates measuring machine (CMM) and video inspection probe and critical dimensions were found within an accuracy of $50 \mu \mathrm{m}$. The actuator was experimentally validated under deployment and handling loads using both a torque meter and a tensile testing machine. Using video inspection probe, the functionality of actuator and accurate measures of the involved displacements were recorded. These measurements proved that the actuator followed the specified sequence with a maximum displacement error of $90 \mu \mathrm{m}$. It was also demonstrated that the ratchet mechanisms worked perfectly, preventing the backlash from the implant and guide system and locking at the end of each sequence. Complete locking of the actuator was proved at the final release stage, thereby preventing the implant from any further deformation post deployment and during release.

It was verified by torque testing that the actuator can withstand an inadvertent force of $520 \mathrm{~N}$ $\mathrm{mm}$ in either a CW or CCW direction. The compressive force applied by the actuator on the implant was validated using tensile testing machine and it was observed that the actuator provides the designed compressive force of $30 \mathrm{~N}$ and $25 \mathrm{~N}$ for the deployment of distal and proximal wings respectively. Fig. 19 shows the behavior of the compressive force applied by the actuator during the deployment phase. Release torque was experimentally evaluated using torque 
measuring equipment. The measured $246.4 \mathrm{~N}$-mm release torque was comparable to theoretically calculated value $242.13 \mathrm{~N}-\mathrm{mm}$ with and accuracy of $1.76 \%$.

In-house in-vitro and mechanical bench testing validated the mechanical behavior and functionality aspects of the actuator. Under microscopic visualization a fabricated actuator was used to deploy implants thereby allowing functionality, mechanical behavior and deployment accuracy to be studied. The wing profile of in-air deployed implants was measured using a video inspection probe. The wing profile of actuator deployed implant was compared with the actually designed wing profile. The standard error of mean of the difference between experimental and simulated profile was 0.003543 and the maximum percent error was found to be $3.129 \%$.

Actuator functionality test for in-vitro usage was performed by deployment of the implant in both porcine tissues and fallopian tubes. The successful deployments in both proved the effectiveness of actuator against the opposing tissue loads. Finally, to validate the actuator in conditions very similar to in-vivo implementation, in-vitro experiments were conducted on explanted uteri. These uteri were removed at hysterectomy for various benign clinical indications. Explanted uteri were chosen as the test model as this is most representative model of the in-vivo situation. The actuator was used for in-vitro deployments of the implant into human explanted uteri. These studies demonstrated that the actuator perfectly deployed the implant in the entire 7 explanted uteri and successfully occluded the fallopian tubes in all uteri.

All testing verified the design of the actuator to successful deploy the implant into a predefined shape to successfully occlude the fallopian tubes resulting in permanent sterilization. 


\section{Conclusions}

The actuator designed and developed in this study proved to be suitable for providing 3 degrees of displacements for deployment and release of an implant. The testing and validation proved that the designed actuator deployed and released an implant with sufficient accuracy and repeatability. The CAD, FEA and optimization were used to properly design the actuator to provide the necessary rotations, translations and worst case loading scenario data of handling loads. The ratchets were evaluated to ensure that they locked correctly and prevented all backlash from the implant, guide tube or guide wire. Furthermore, failure during any inadvertent forces was also investigated and analysed. It was also demonstrated that the actuator secured the implant after deployment by completely locking itself before release. The actuator was successfully tested over a wide range of operating conditions and under all possible loading conditions. Further development work is underway in preparation for "first-in-man” clinical trials for the purpose of human factor safety. It is envisaged that the final actuator design will be cost effective, easy to use and will be both practical and reproducible. In addition, the concept of this actuator could be applicable to deploy various other medical devices and could be implemented into devices requiring rotational and translations movements.

\section{.Acknowledgements}

The authors thank Vasorum /Alta Science team for their support. The authors also thank Enterprise Ireland for the financial support for this project. 


\section{References}

1. Cooper, J. M., Carignan, C. S., Cher, D. and Kerin, J. F., Microinsert nonincisional hysteroscopic sterilization, Obstet. Gynecol., 102, 59-67 (2003)

2. Abbott, J., Transcervical sterilization, Best Pract. Res. Clin. Obstet. Gynaecol., 19, 743756 (2005)

3. Brumsted, J. R., Shirk, G., Soderling, M. J. and Reed, T., Attempted transcervical occlusion of the fallopian tube with the Nd:YAG laser, Obstet. Gynecol., 77, 327-328 (1991)

4. Donnez, J., Malvaux, V., Nisolle, M. and Casañas, F., Hysteroscopic sterilization with the Nd:YAG laser, J. Gynecol. Surg., 6, 149-153 (1990)

5. Carr-Brendel, V. E., Stewart, D. R., Harrington, D. C., Leal, J. G. G. and Vancaillie, T., A new transcervical sterilization procedure: results of a pilot implant study in humans, Obstet. Gynecol., 97, 8 (2001)

6. Carr-Brendel, V. E., Stewart, D. R., Harrington, D. C., Dhaka, V. K., Breining, P. M. and Vancaillie, T., A new transcervical sterilization procedure-6-month preclinical results, Obstet. Gynecol., 97, 15-16 (2001)

7. McSwain, H. and Brodie, M. F., Fallopian Tube Occlusion, An Alternative to Tubal Ligation, Tech. Vasc. Interv. Radiol., 9, 24-29 (2006)

8. Ubeda, A., Labastida, R. and Dexeus, S., Essure ${ }^{\circledR}$ : a new device for hysteroscopic tubal sterilization in an outpatient setting, Fertil. Steril., 82, 196-199 (2004)

9. Grunwald, A., Olabi, A.G., Design of a magnetostrictive (MS) actuator, Sensors and Actuators A, 144, 161-175 (2008) 
10. Grunwald, A., Olabi, A.G., Computation of magnetic field in an actuator, Simulation Modelling Practice and Theory, 16, 1728-1736 (2008)

11. Rehan, M. and Olabi, A. G., Novel Implant for Transcervical Sterilization, J. Biosci. Bioeng., doi:10.1016/j.jbiosc.2010.02.002 (2010)

12. Rehan, M. and Olabi, A. G., Automated CAD System Based Design Optimization Methodology, p. 377-385. In Simson, R. (ed.), Manufacturing and Design: The Next Generation. Proceeding of the $25^{\text {th }}$ International Manufacturing Conference. Dublin Institute of Technology, Dublin, Ireland (2008)

13. Rehan, M. and Olabi, A. G., Parametric Design Optimization By Integrating CAD Systems And Optimization Tools, V. J. Segui and M. J. Reig (ed.), CPl 181, Third Manufacturing Engineering Society International Conference, American Institute of Physics, 681-692 (2009)

14. Kong, Yong-Ku, Lowe B. D., Optimal cylindrical handle diameter for grip force tasks, International Journal of Industrial Ergonomics, 35, 495-507 (2005)

15. Kong, Yong-Ku, Lowe B. D., Evaluation of handle diameters and orientations in a maximum torque task, International Journal of Industrial Ergonomics, 35, 1073-1084 (2005)

16. Knut O. Kverneland, Screw Threads, Chapter 8, Metric Standards for Worldwide Manufacturing, ASME Press, New York, pp. 131-140, (1996)

\section{Bryan Wimer, Ren G. Dong, Daniel E. Welcome, Christopher Warren, Thomas W.} McDowell, Development of a new dynamometer for measuring grip strength applied on a cylindrical handle, Medical Engineering \& Physics, 31, 695-704 (2009) 


\section{List of Figures}

Figure 1. Deployed Implant at left Fallopian tube using Actuator ......................................................26

Figure 2. Micrograph of the implant pre-deployment and post-deployment .............................................2 27

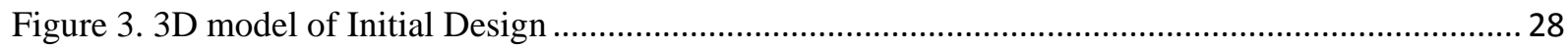

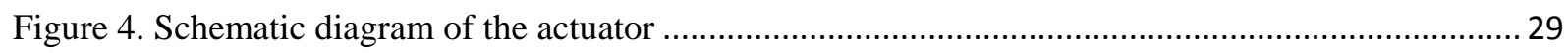

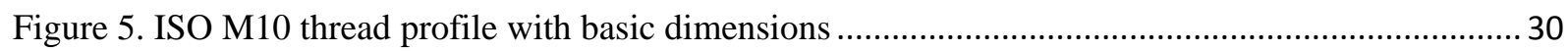

Figure 6. Force balance diagram of a standard ISO thread ...................................................................... 31

Figure 7. Operational Sequence of Actuator (a) Initial Condition (b) Counter-Clockwise Rotation followed by Spring Compression (c) Clockwise Rotation followed by Spring Compression (d) Clockwise Rotation of Release Knob

Figure 8. Optimization of Fore-body (a) Applied Boundary Conditions (b) FE Mesh $\quad$ (c) von-Mises Stress Distribution (d) Total deformation 33

Figure 9. Optimization of Handle-body (a) Applied Boundary Conditions (b) FE Mesh $\quad$ (c) von-Mises Stress Distribution (d) Total deformation..... 34

Figure 10. Optimization of Ratchet Mechanism (a) Applied Boundary Conditions (b) FE Mesh (c) vonMises Stress Distribution (d) Total deformation.

Figure 11. Release Mechanism (a) Applied Boundary Conditions (b) FE Mesh (c) von-Mises Stress Distribution (d) Total deformation.....

Figure 12. Fabricated Stainless Steel Actuator (a) Assembled (b) Exploded..... 37

Figure 13. Torque Measuring Setup for Torque measurement on (a) Handle-body (b) Release Knob (c) Release tube gripped inside actuator...... 38

Figure 14. Testing setup for compression load measurements ……......................................................... 39

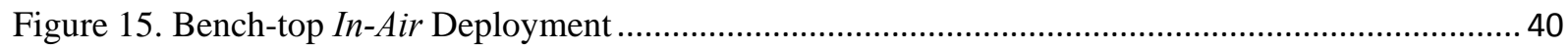

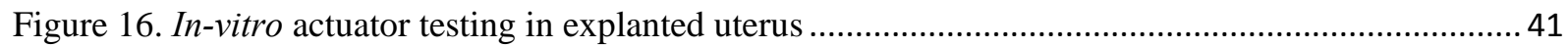

Figure 17: Mesh Convergence (a) Fore-body (b) Handle-body (c) Ratchet Mechanism (d) Release Mechanism

Figure 18. Design variable response vs. von-Mises stress distribution ................................................. 43

Figure 19. Actuator Compressive Force Characteristic Curve …................................................................ 44 


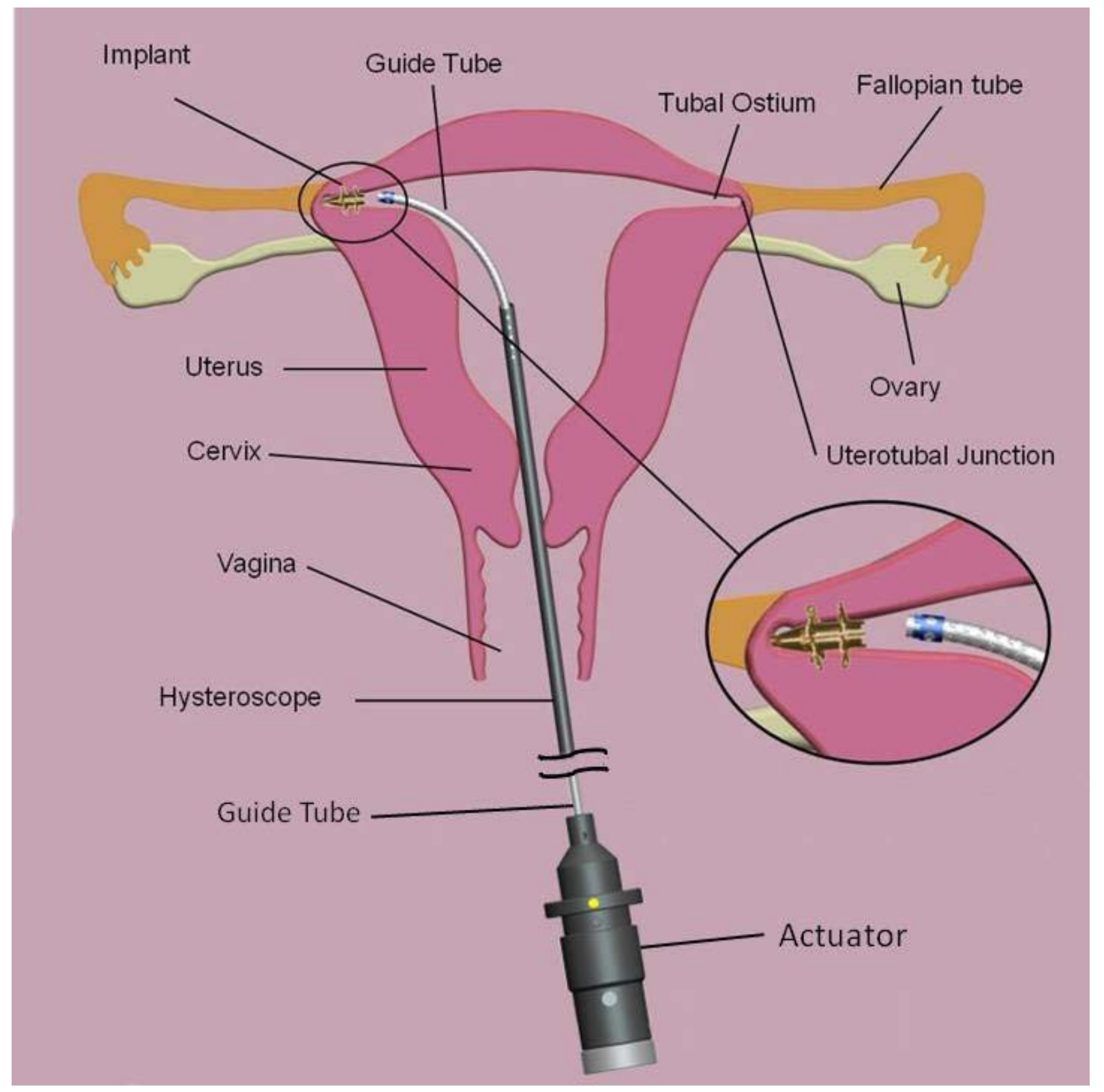

Figure 1. Deployed Implant at left Fallopian tube using Actuator 


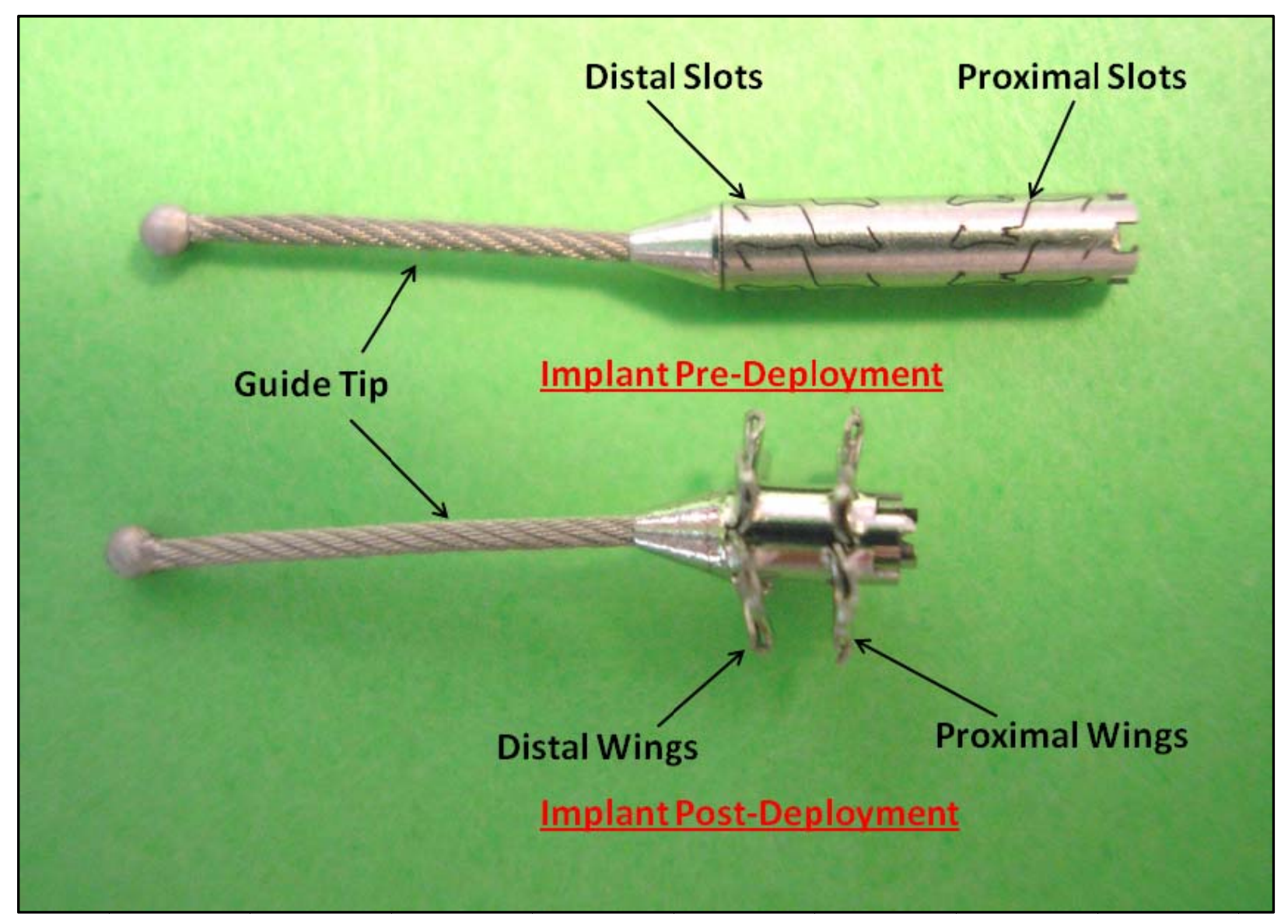

Figure 2. Micrograph of the implant pre-deployment and post-deployment 


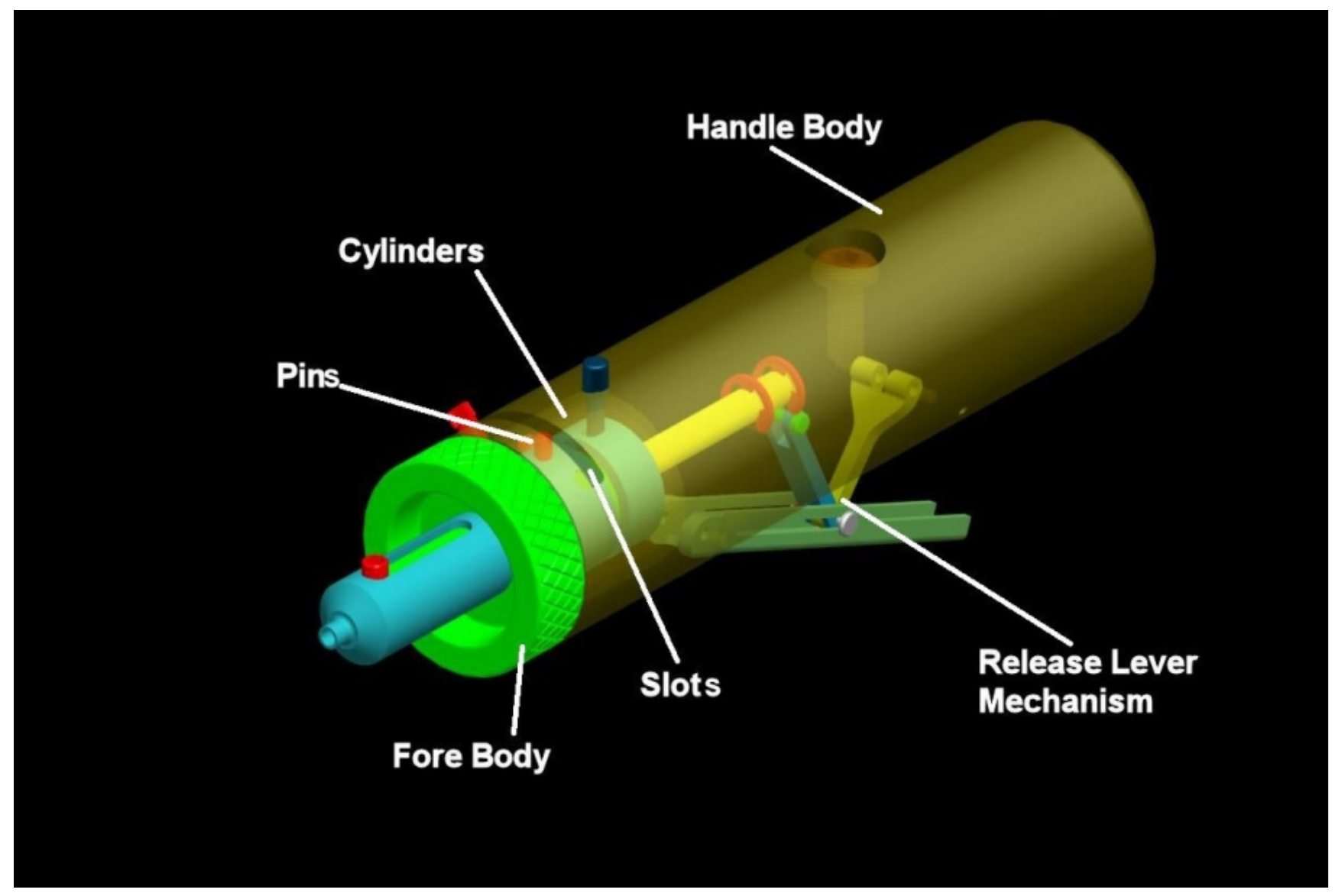

Figure 3. 3D model of Initial Design 


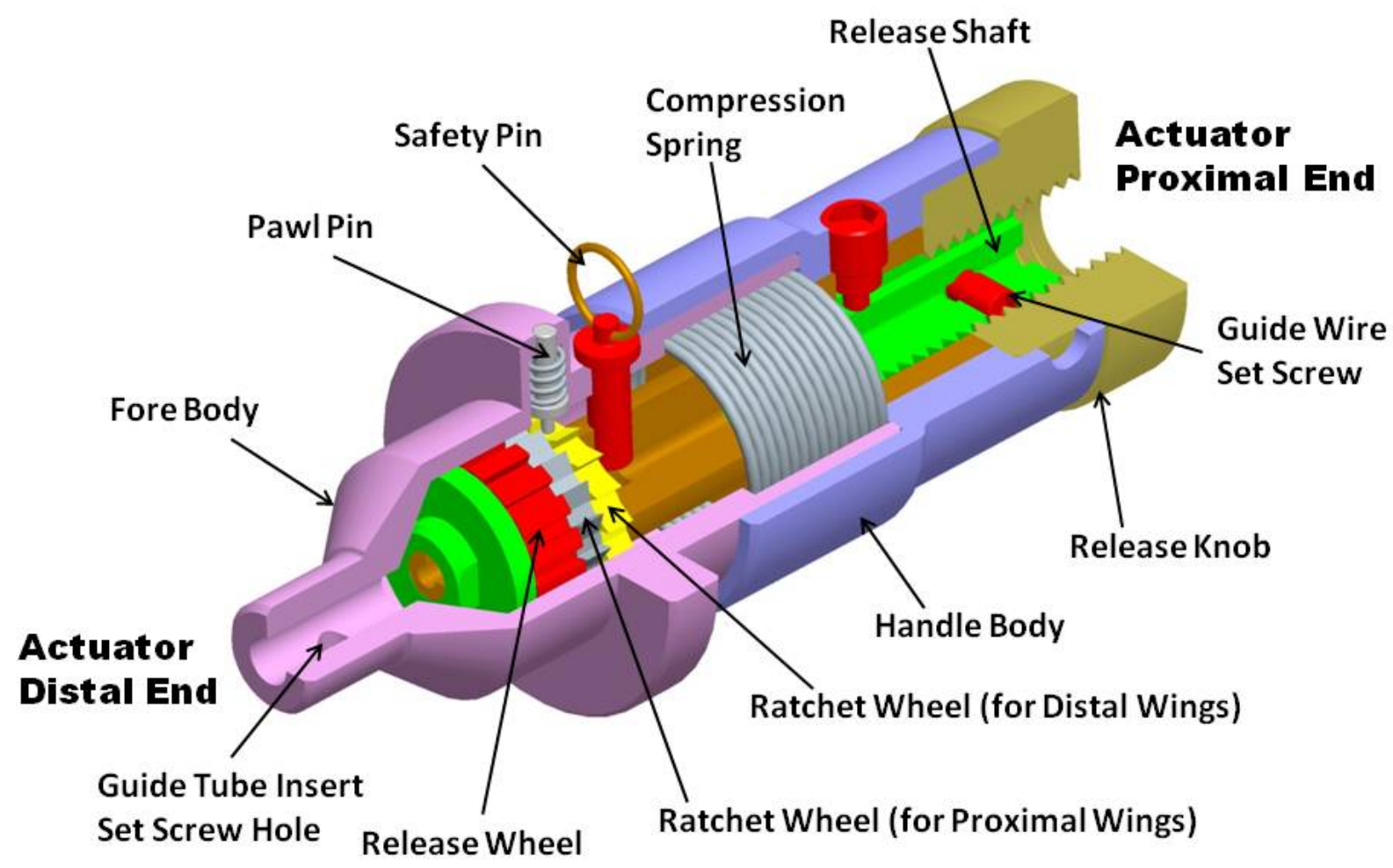

Figure 4. Schematic diagram of the actuator 


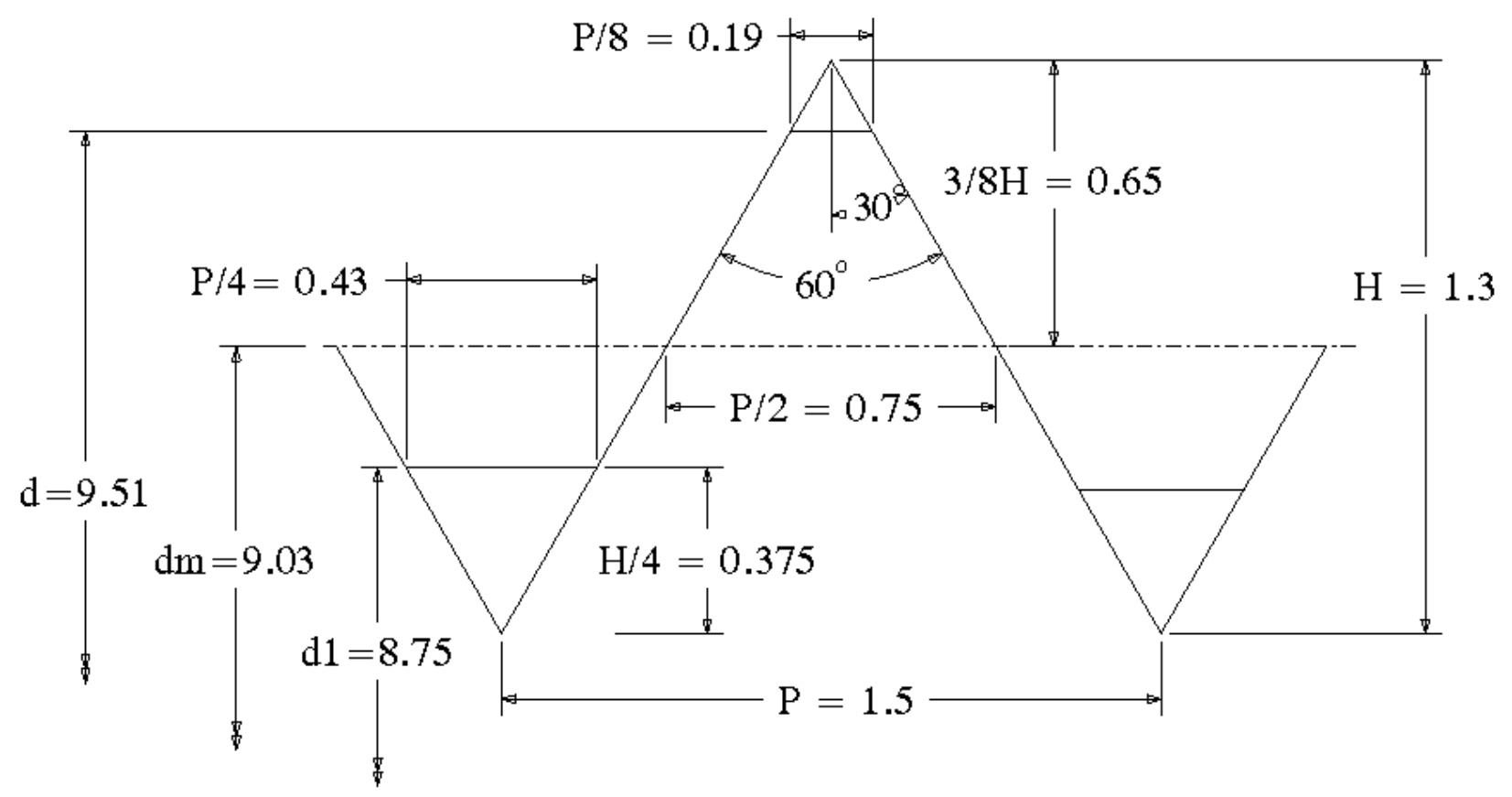

Figure 5. ISO M10 thread profile with basic dimensions 


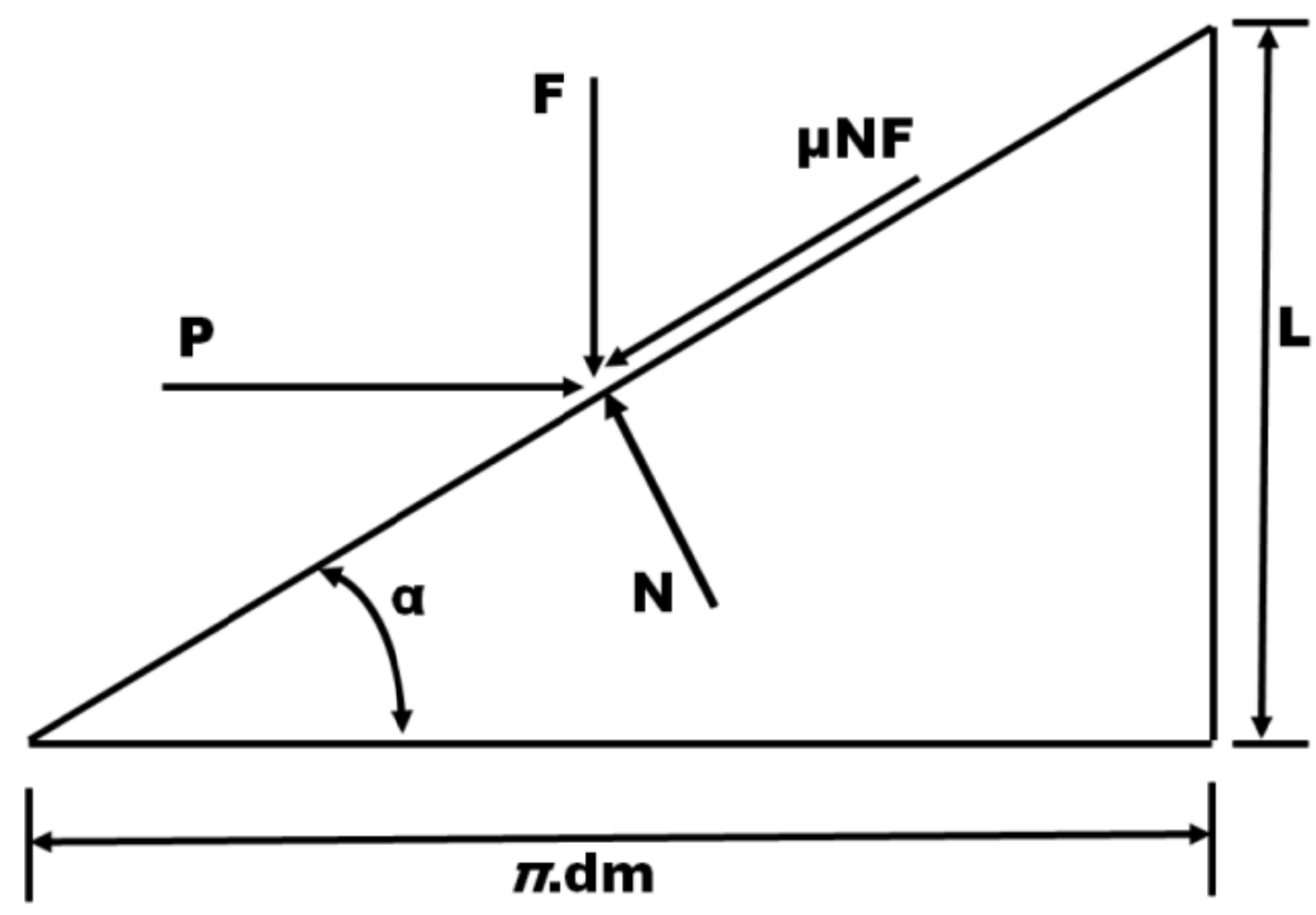

Figure 6. Force balance diagram of a standard ISO thread 


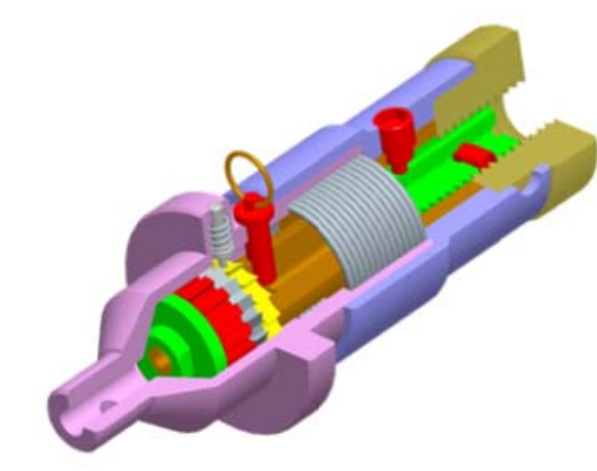

(a)

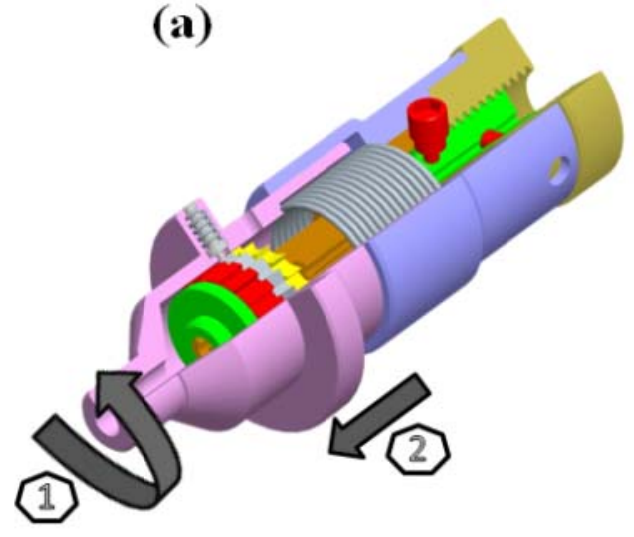

(c)

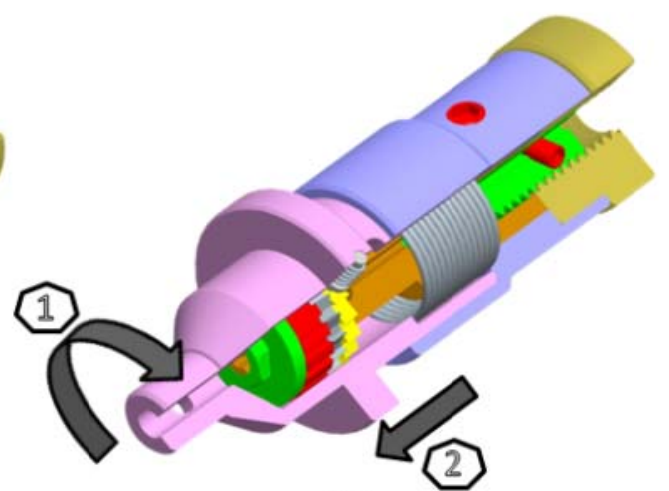

(b)

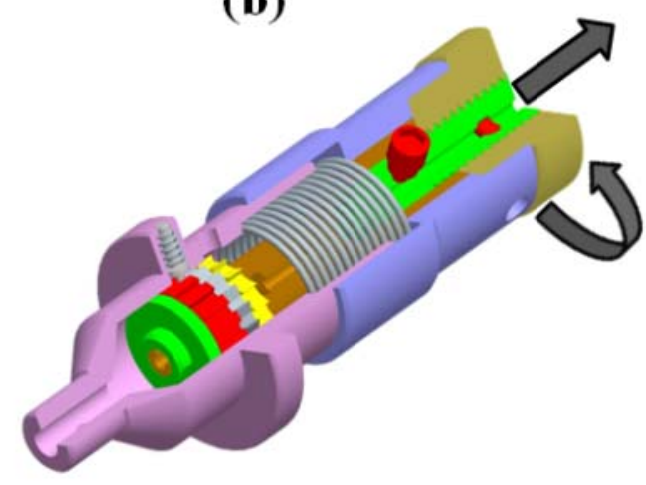

(d)

Figure 7. Operational Sequence of Actuator (a) Initial Condition (b) Counter-Clockwise Rotation followed by Spring Compression (c) Clockwise Rotation followed by Spring Compression (d) Clockwise Rotation of Release Knob 


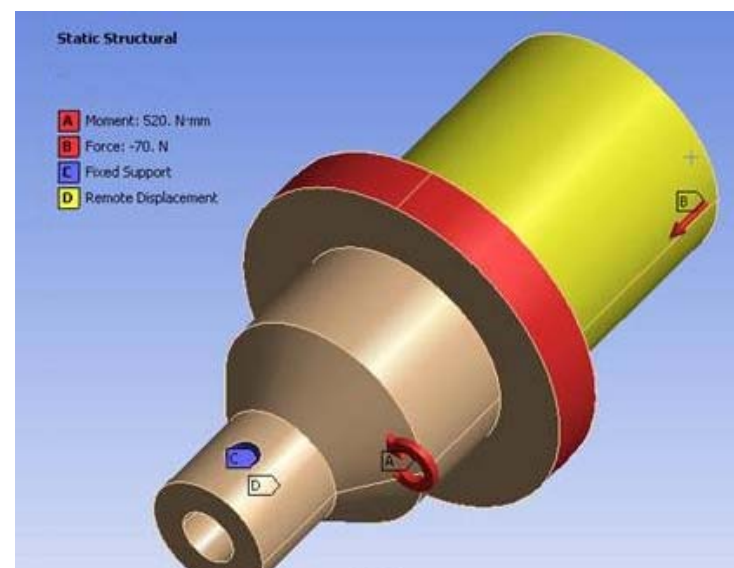

(a)

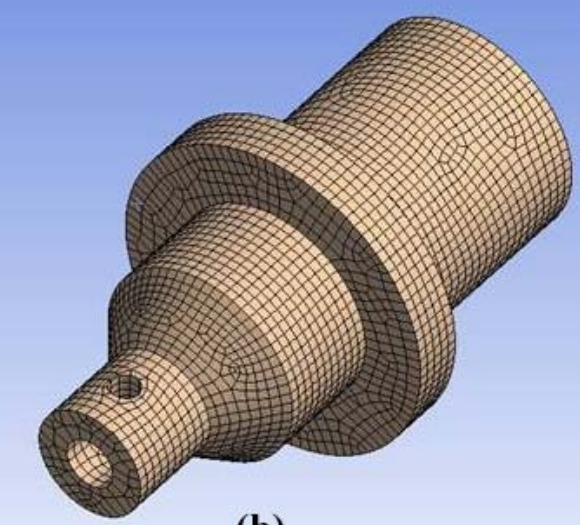

(b)

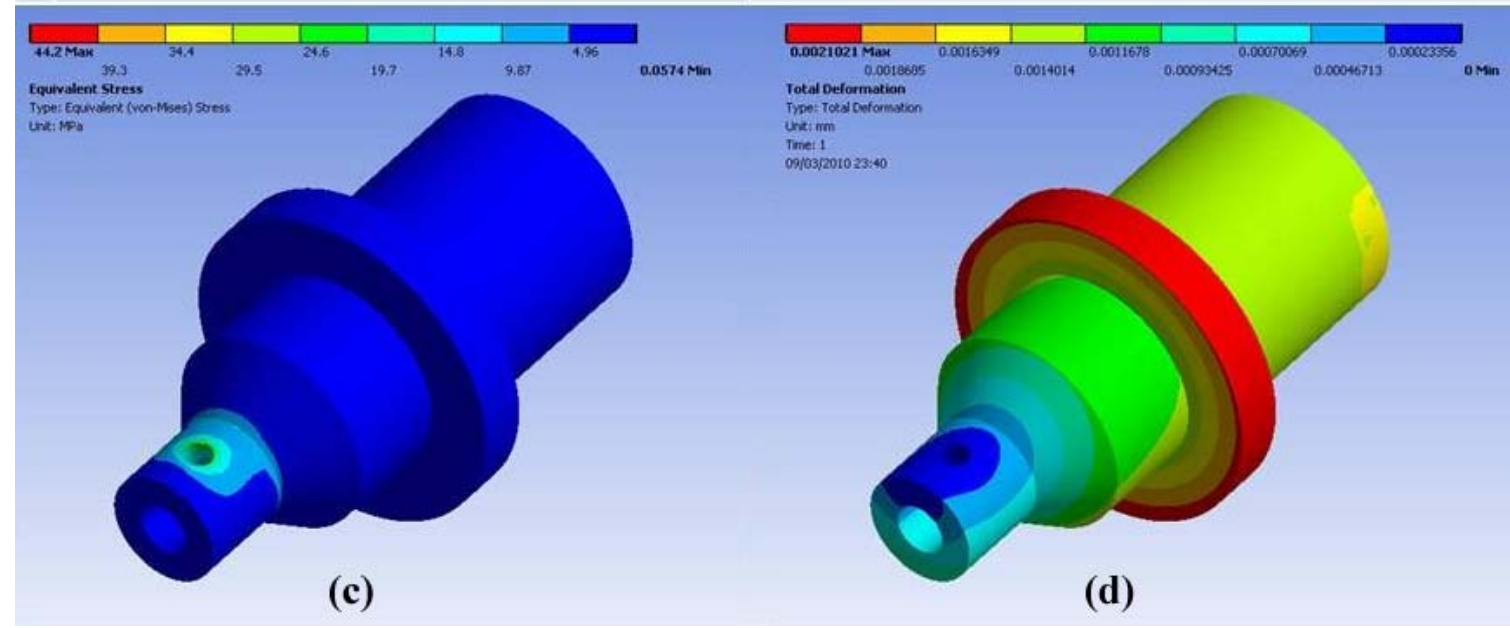

Figure 8. Optimization of Fore-body (a) Applied Boundary Conditions (b) FE Mesh (c) von-Mises Stress Distribution (d) Total deformation 


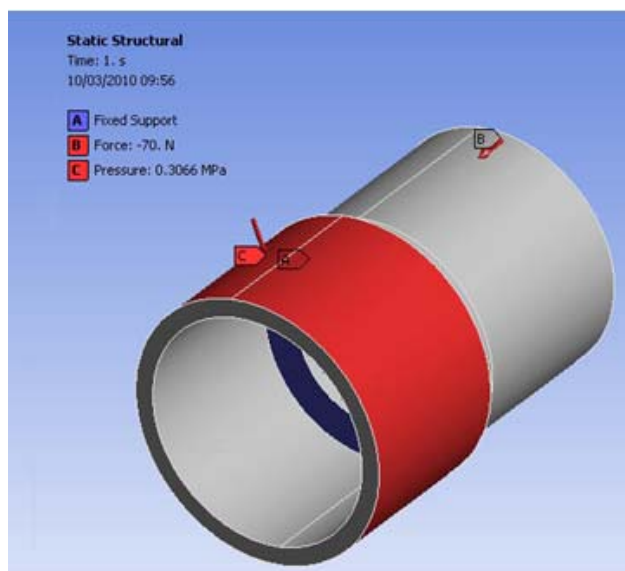

(a)

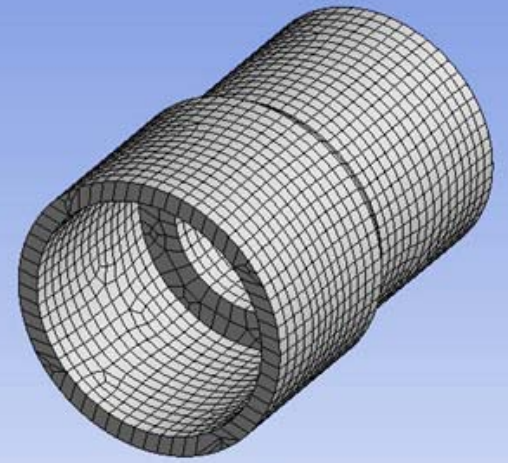

(b)

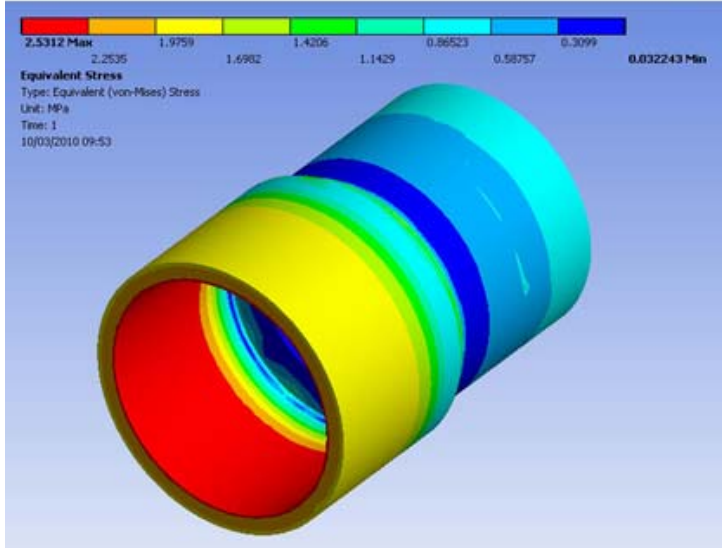

(c)

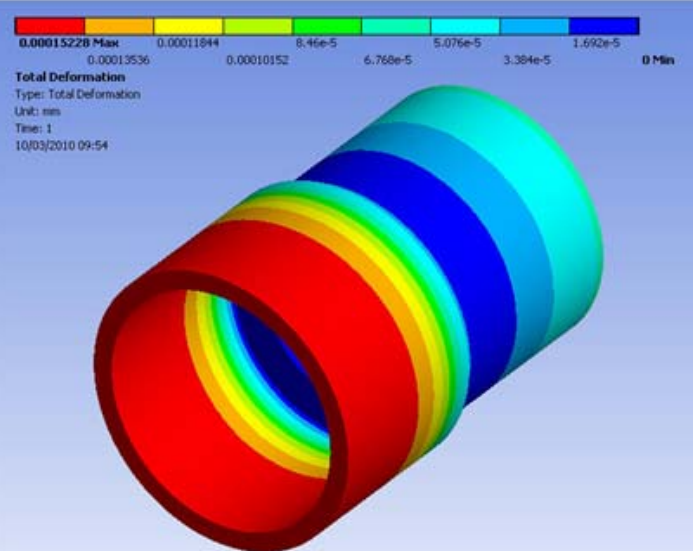

(d)

Figure 9. Optimization of Handle-body (a) Applied Boundary Conditions (b) FE Mesh (c) von-Mises Stress Distribution (d) Total deformation 


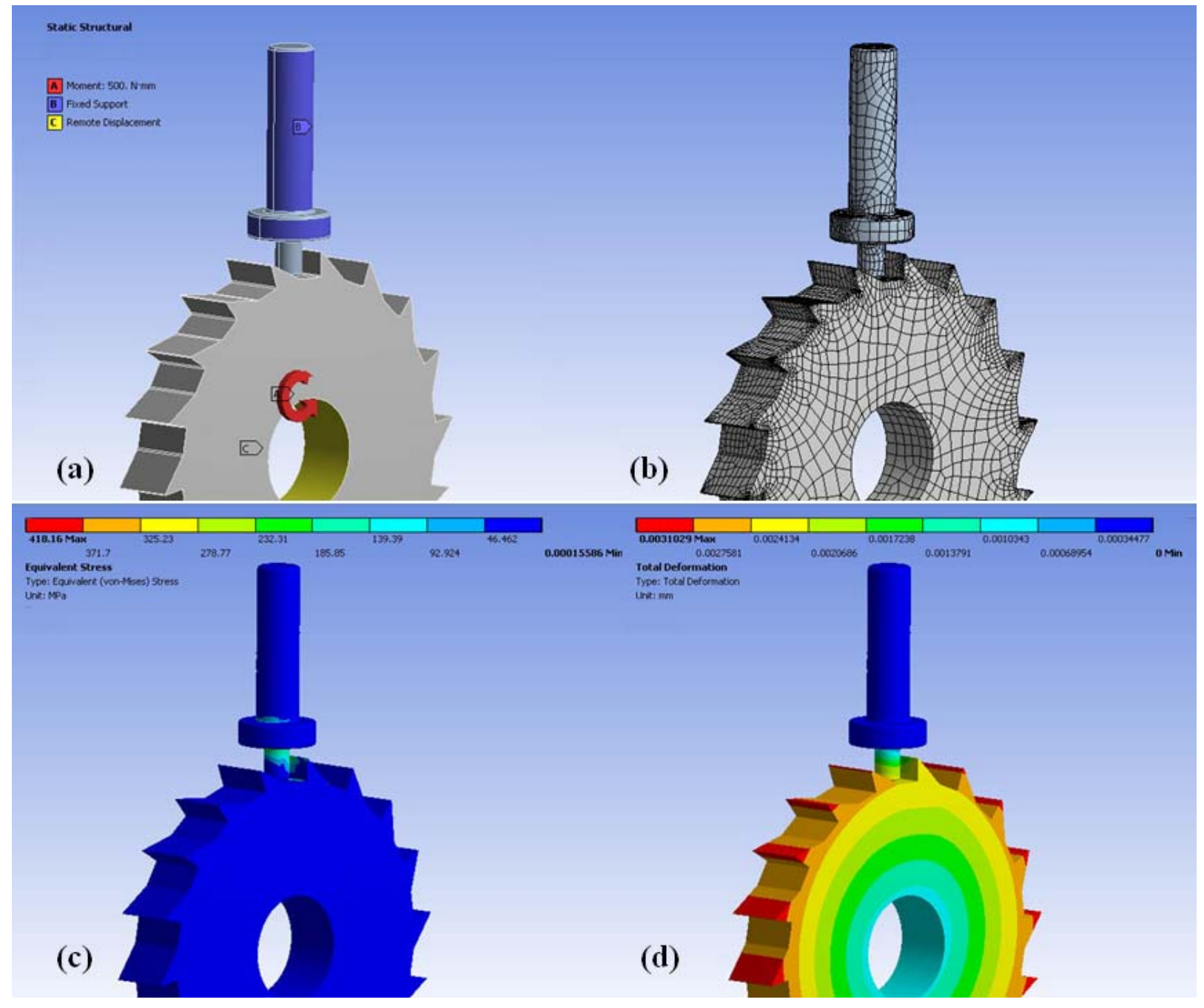

Figure 10. Optimization of Ratchet Mechanism (a) Applied Boundary Conditions (b) FE Mesh (c) von-Mises Stress Distribution (d) Total deformation 


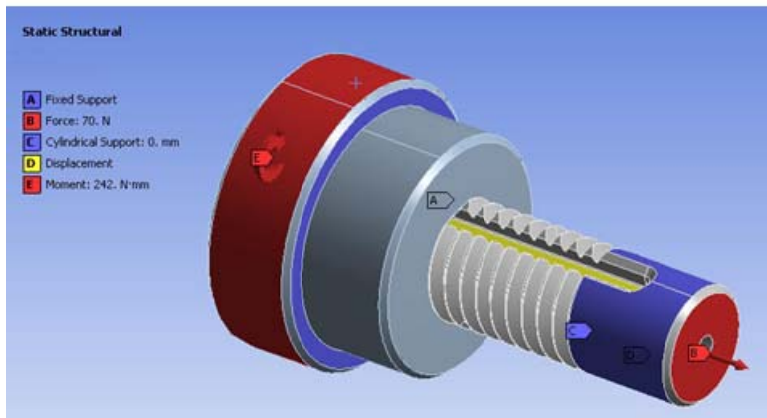

(a)

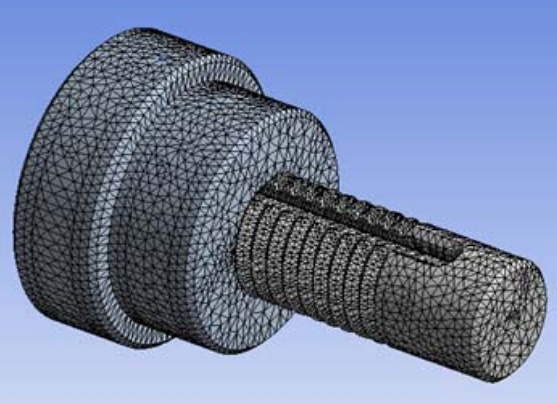

(b)

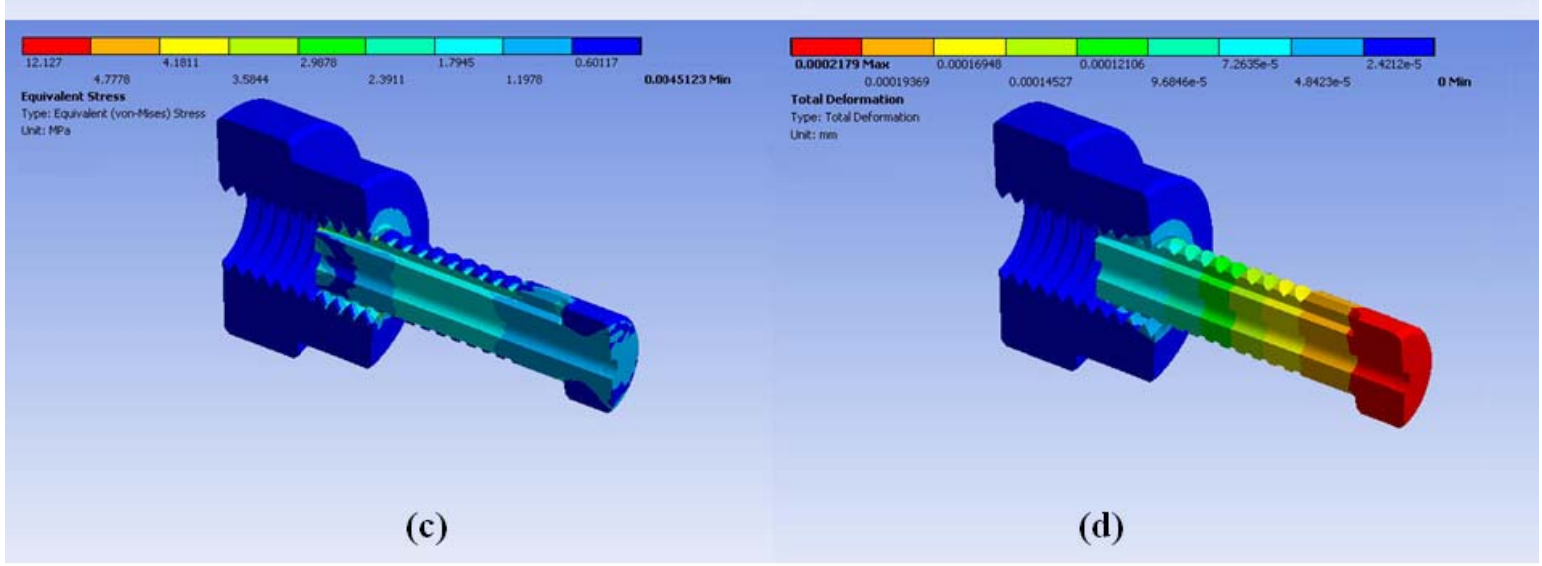

Figure 11. Release Mechanism (a) Applied Boundary Conditions (b) FE Mesh (c) vonMises Stress Distribution (d) Total deformation 


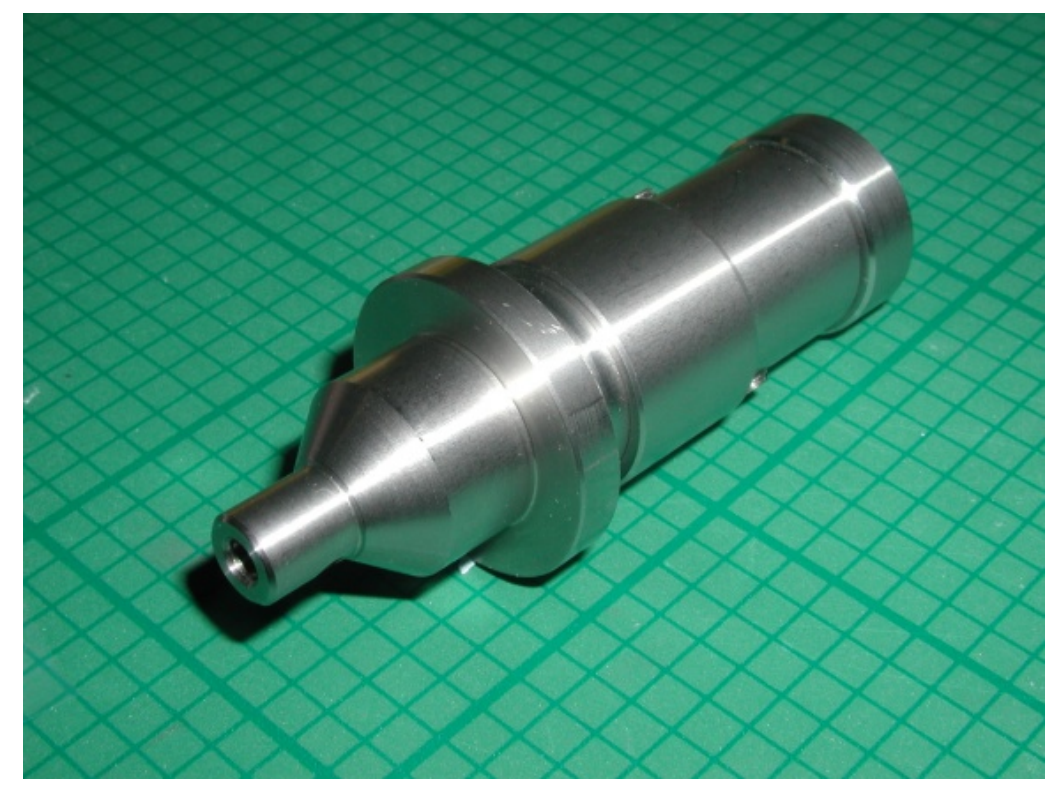

(a)

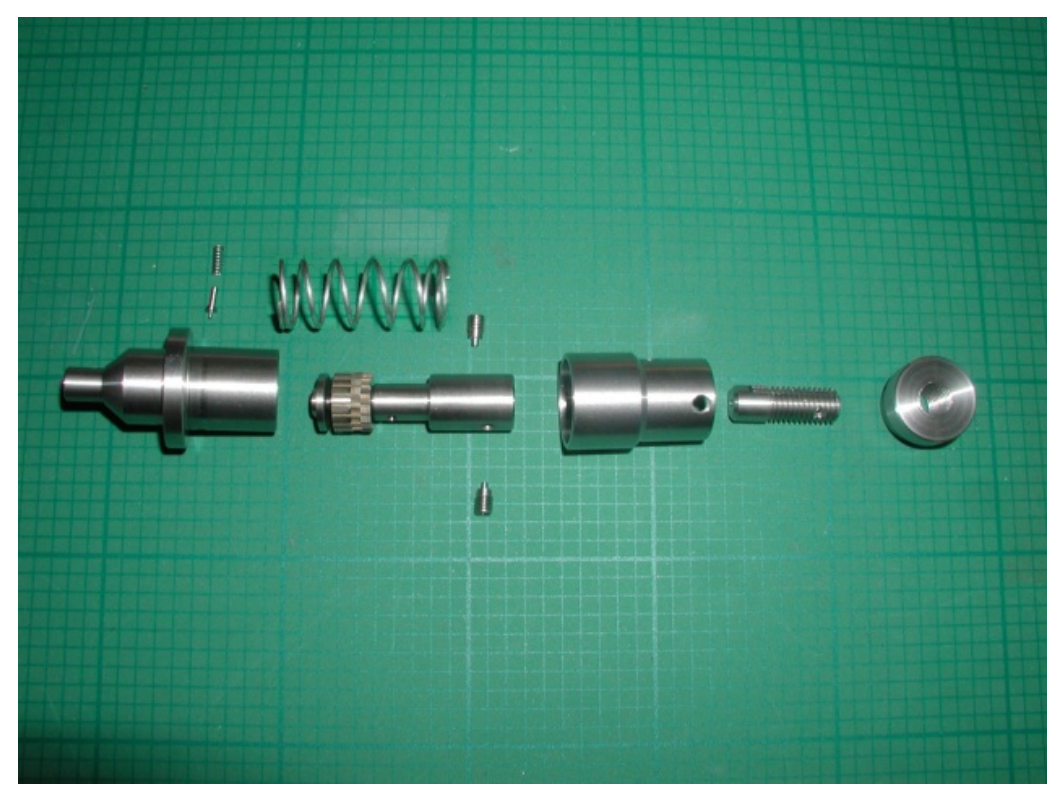

(b)

Figure 12. Fabricated Stainless Steel Actuator (a) Assembled (b) Exploded 

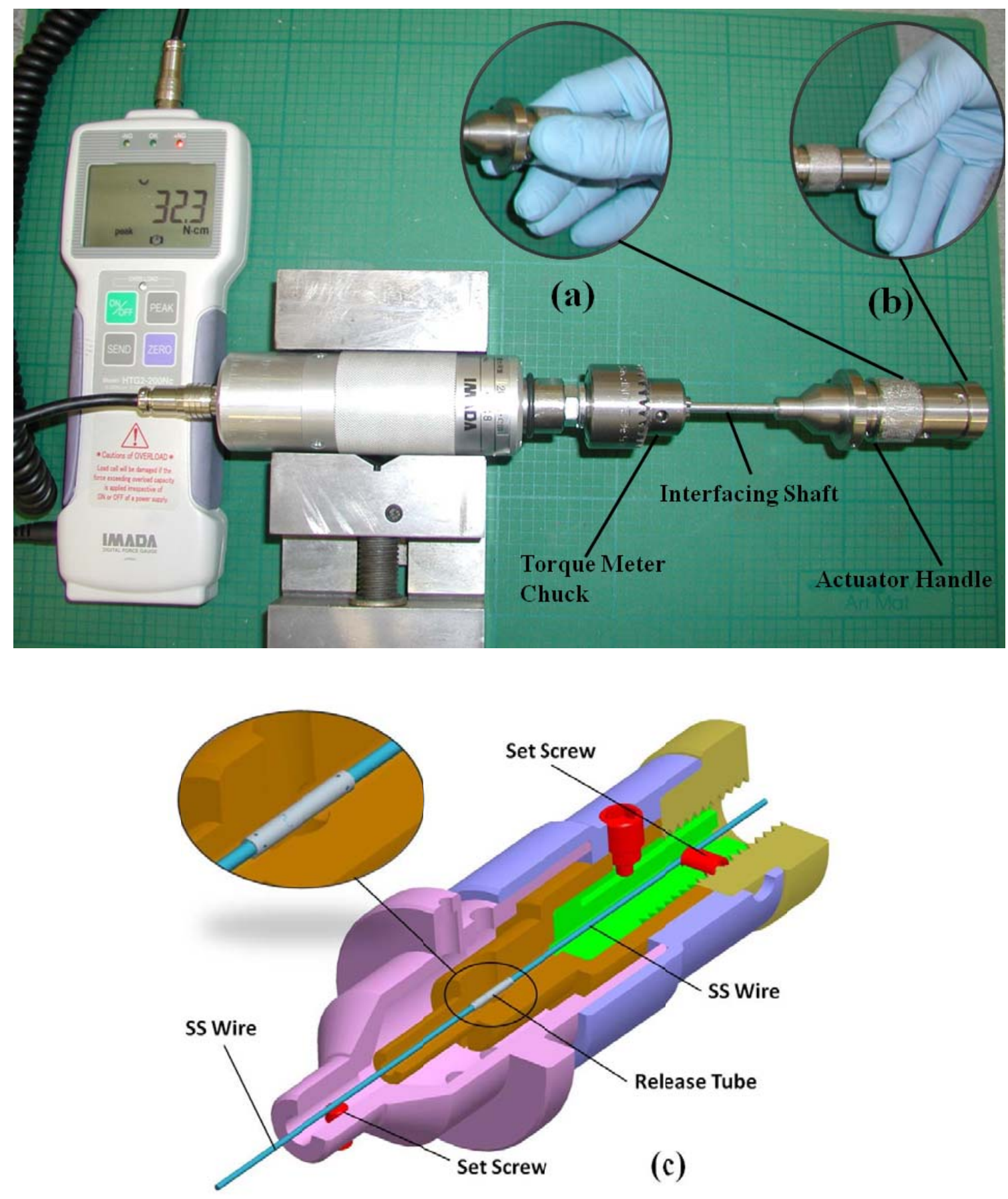

Figure 13. Torque Measuring Setup for Torque measurement on (a) Handle-body (b) Release Knob (c) Release tube gripped inside actuator 


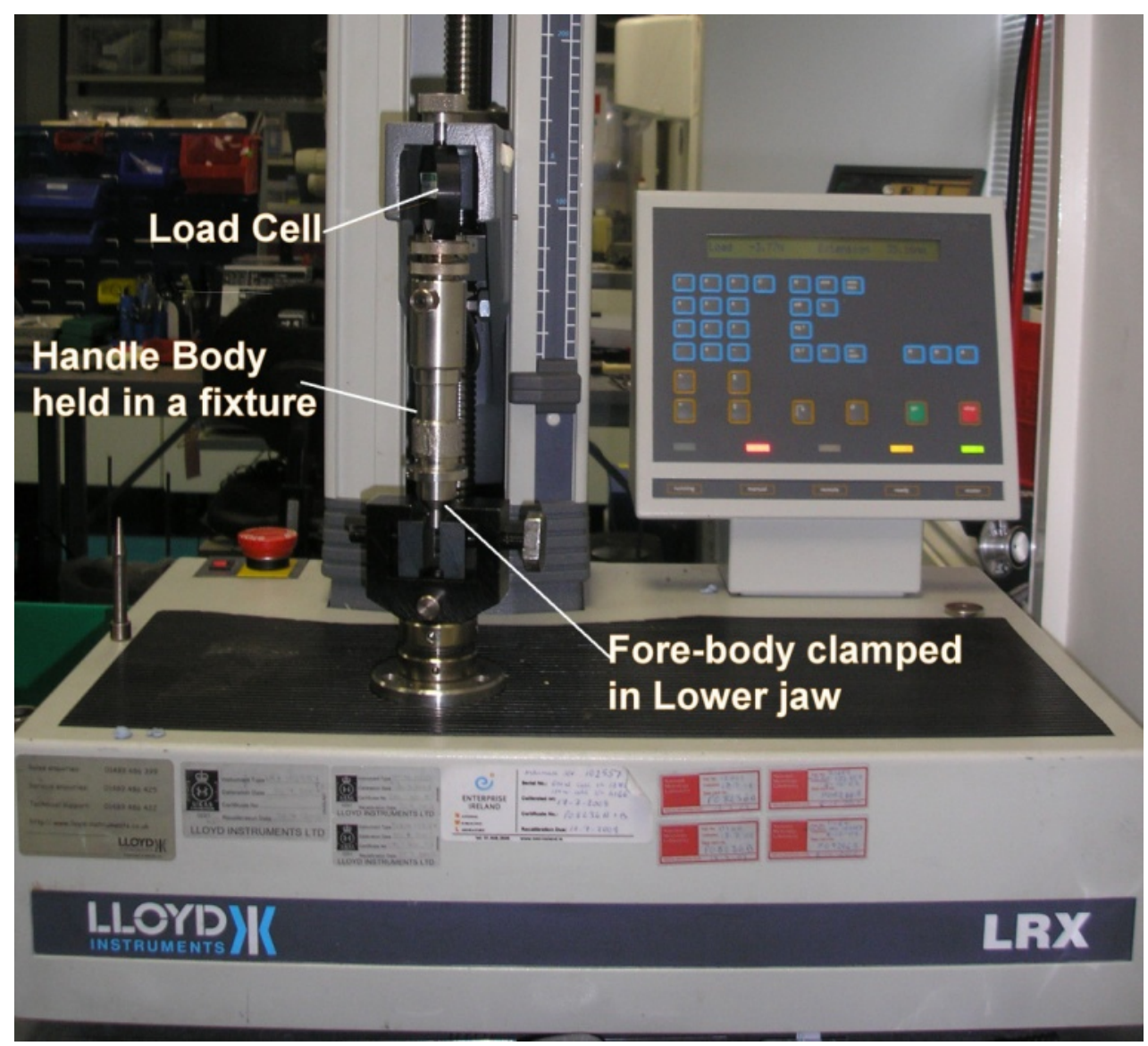

Figure 14. Testing setup for compression load measurements 


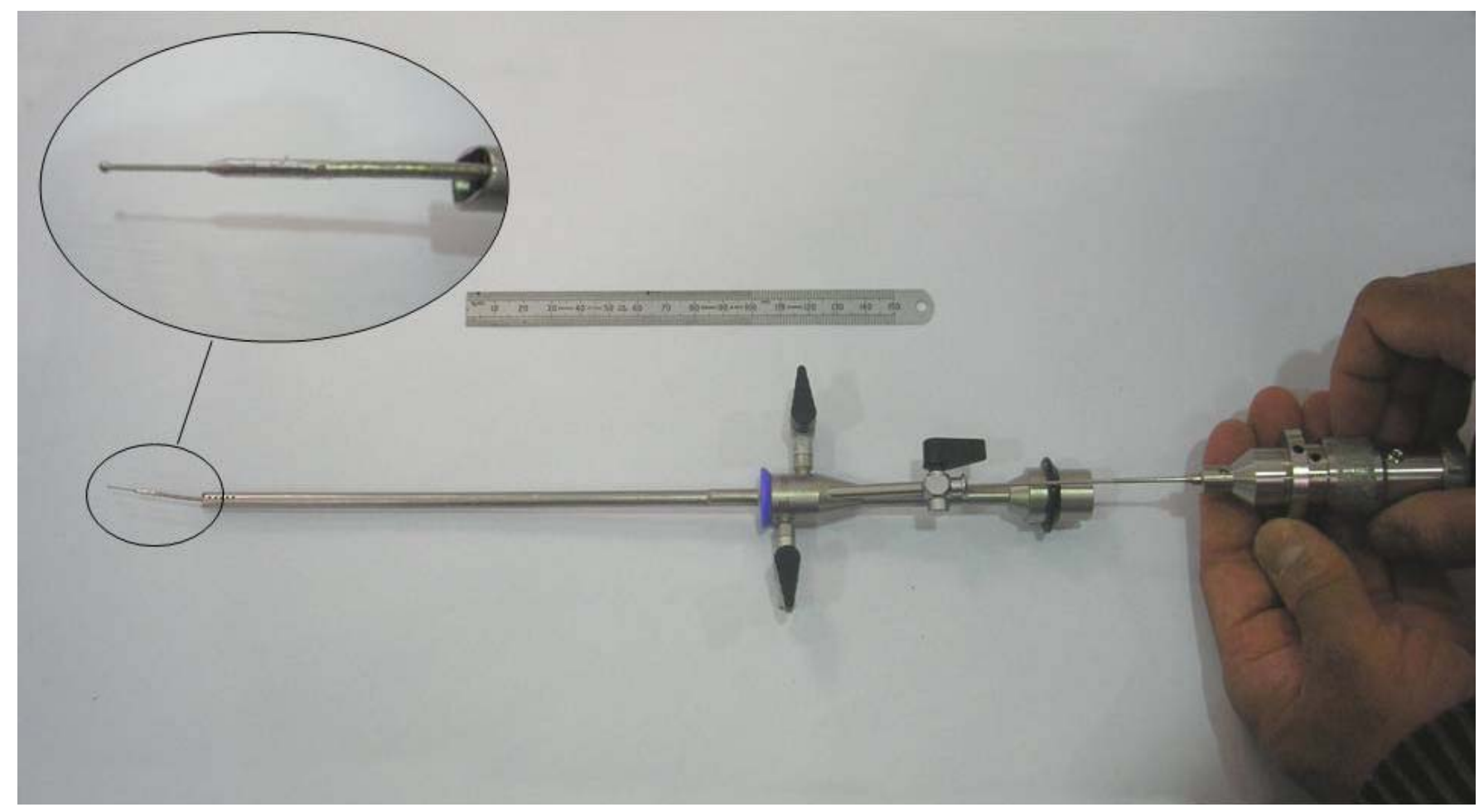

Figure 15. Bench-top In-Air Deployment 


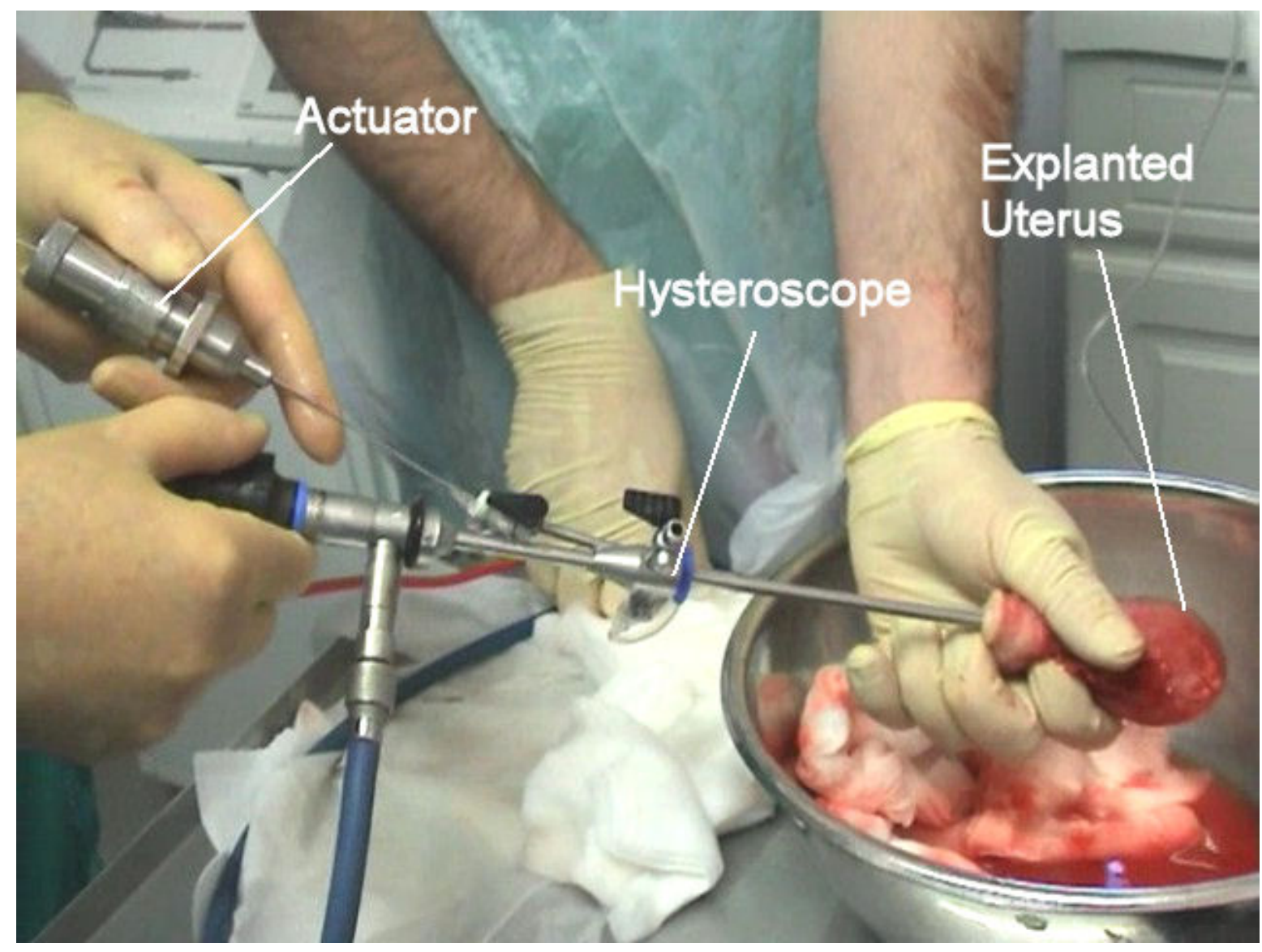

Figure 16. In-vitro actuator testing in explanted uterus 


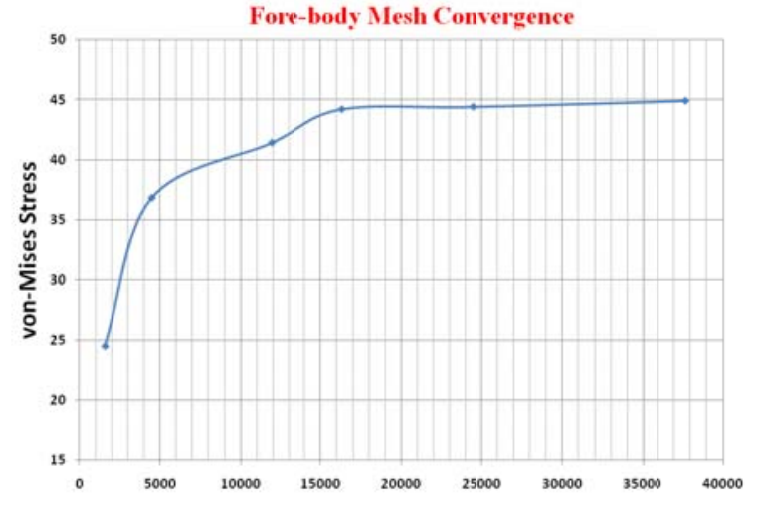

(a)

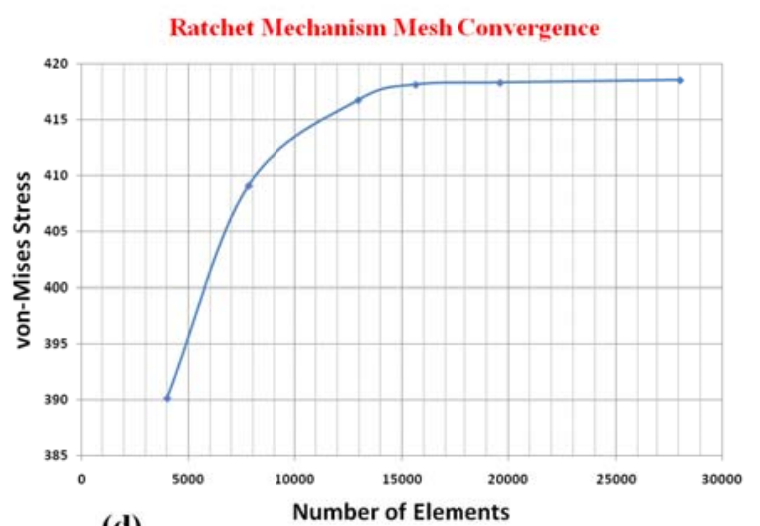

(d)

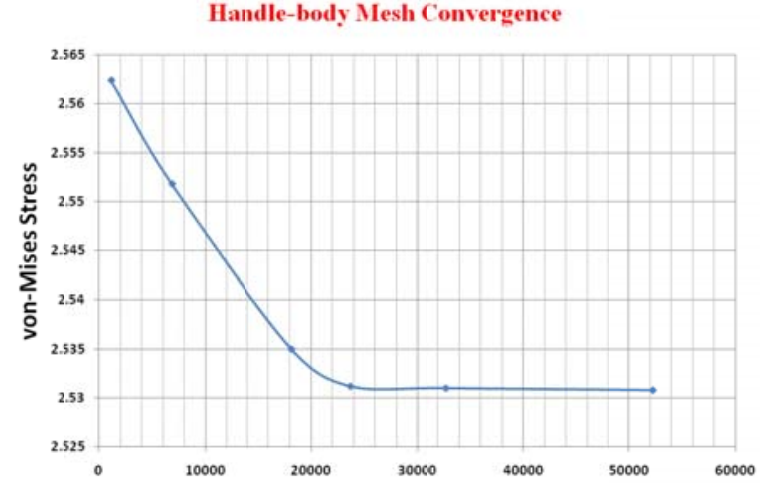

(b)

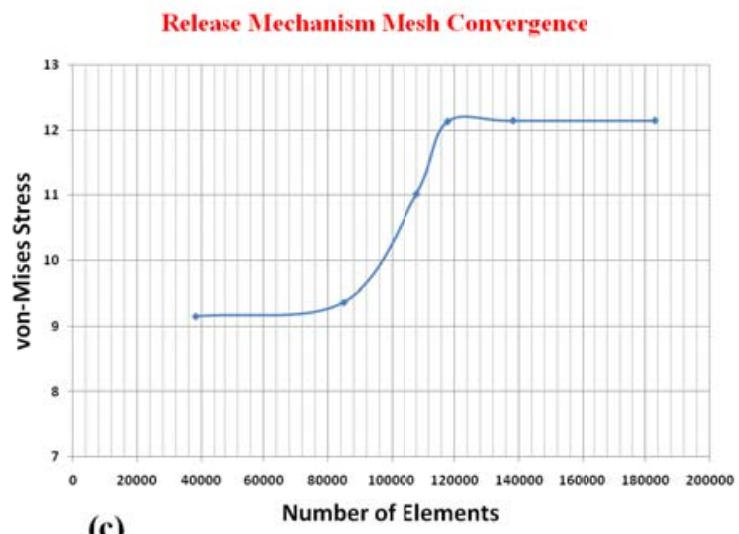

(c)

Figure 17: Mesh Convergence (a) Fore-body (b) Handle-body (c) Ratchet Mechanism (d) Release Mechanism 


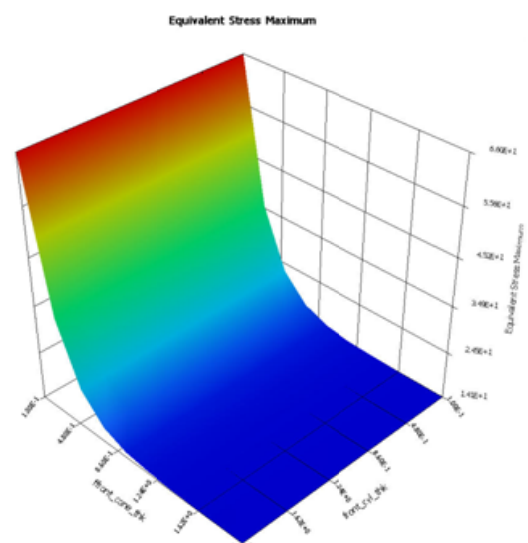

(a)

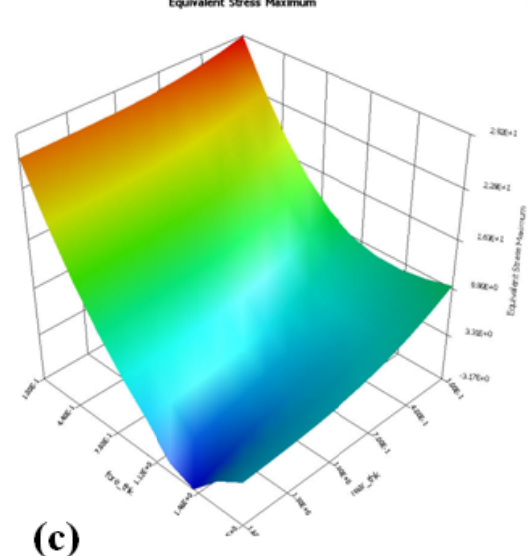

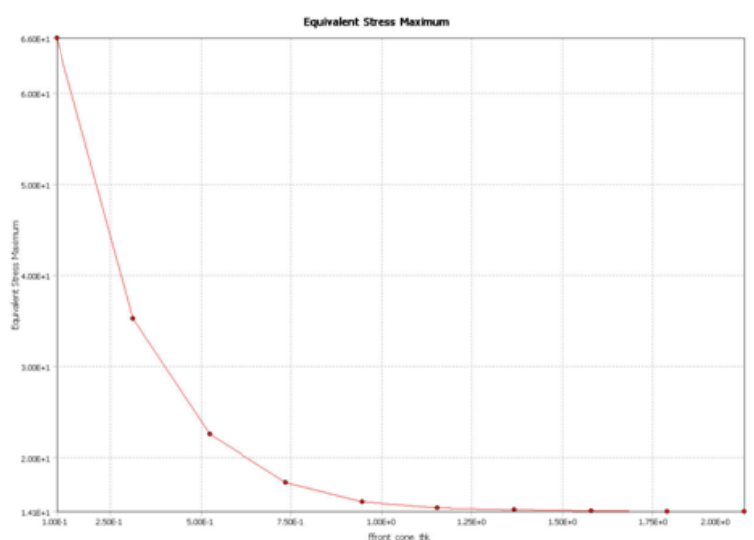

(b)

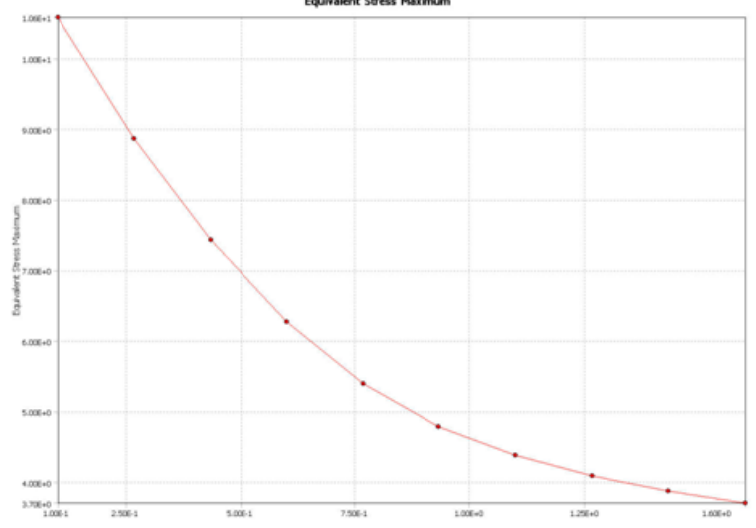

(d)

Figure 18. Design variable response vs. von-Mises stress distribution 


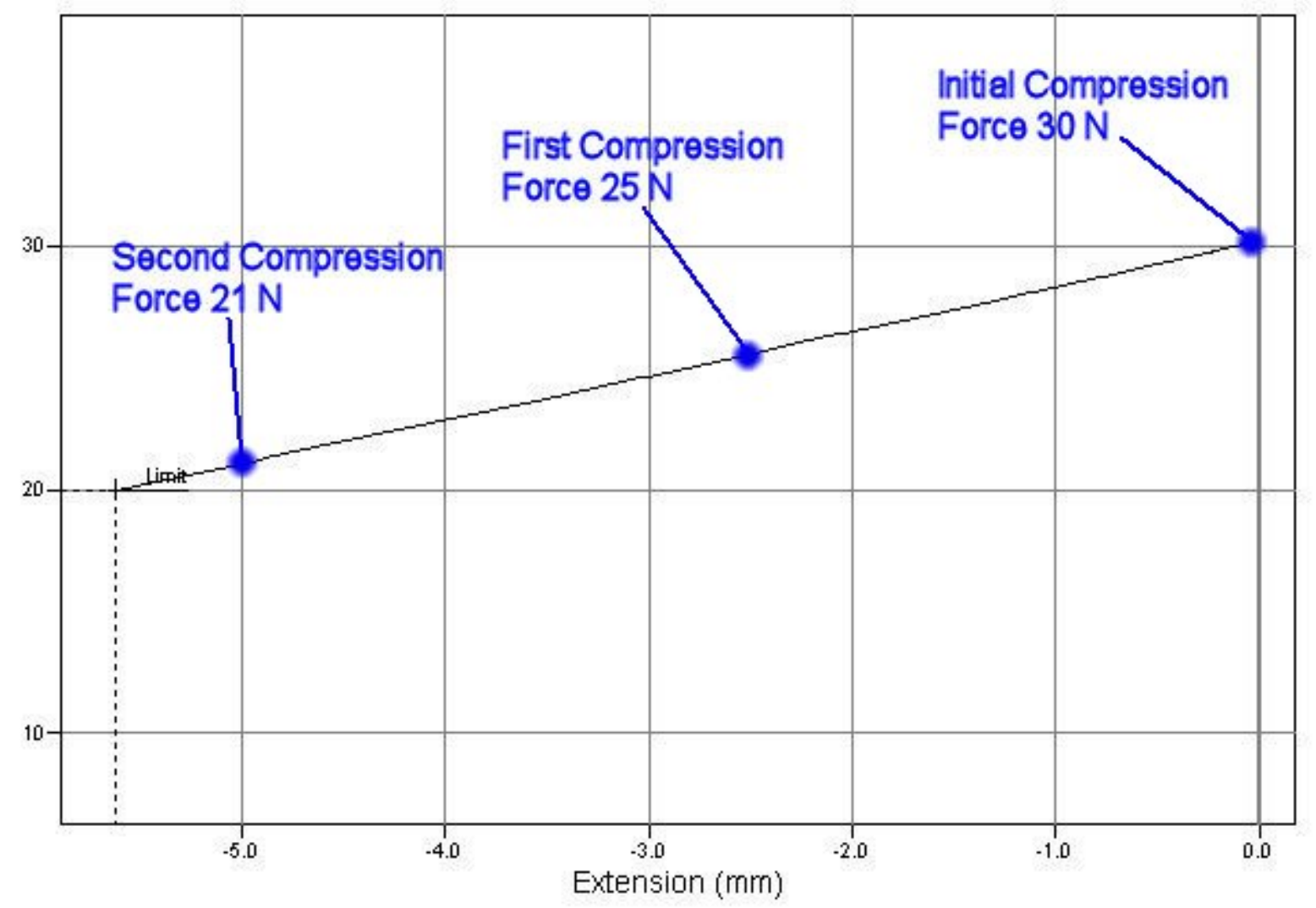

Figure 19. Actuator Compressive Force Characteristic Curve 


\section{List of Table}

Table I. Actuator Handling and Operational Loads.......................................45

Table II. Mesh Convergence Data for Actuator Components..............................46

Table I. Actuator Handling and Operational Loads

\begin{tabular}{|l|l|c|c|c|c|}
\hline \multirow{2}{*}{ Components } & \multicolumn{2}{|c|}{ Deployment and Release Loads } & \multicolumn{2}{c|}{ Handling Loads } \\
\cline { 3 - 6 } & $\begin{array}{c}\text { Compression / } \\
\text { Tension (N) }\end{array}$ & $\begin{array}{c}\text { Torque } \\
(\mathrm{N}-\mathrm{mm})\end{array}$ & Grip Force (N) & $\begin{array}{c}\text { Torque } \\
(\mathrm{N}-\mathrm{mm})\end{array}$ \\
\hline Handle-body & 70 & $15.4,16$ & 826 & 520 \\
\hline Fore-body & $25,30,70$ & $15.4,16$ & 0 & 520 \\
\hline \multirow{2}{*}{$\begin{array}{l}\text { Ratchet } \\
\text { Mechanism }\end{array}$} & Ratchet Wheel & 0 & 0 & 0 & 520 \\
\cline { 2 - 6 } $\begin{array}{l}\text { Release } \\
\text { Mechanism }\end{array}$ & Release Knob & 70 & 320 & 826 & 520 \\
\cline { 2 - 6 } & Release Shaft & 70 & $15.4,16$ & 0 & 0 \\
\hline
\end{tabular}


Table II. Mesh Convergence Data for Actuator Components

\begin{tabular}{|c|c|c|c|c|}
\hline Model & Mesh & $\begin{array}{c}\text { Number of } \\
\text { Nodes }\end{array}$ & $\begin{array}{c}\text { Number of } \\
\text { Elements }\end{array}$ & $\begin{array}{c}\text { Von-Mises } \\
\text { Stress (MPa) }\end{array}$ \\
\hline \multirow{6}{*}{ Fore-body } & $\mathrm{A}$ & 5115 & 1580 & 24.5 \\
\hline & $\mathrm{B}$ & 16309 & 4483 & 36.8 \\
\hline & $\mathrm{C}$ & 40979 & 11925 & 41.4 \\
\hline & $\mathrm{D}$ & 58663 & 16313 & 44.2 \\
\hline & $\mathrm{E}$ & 85139 & 24457 & 44.4 \\
\hline & $\mathrm{F}$ & 127873 & 37711 & 44.9 \\
\hline \multirow{6}{*}{ Handle-body } & $\bar{A}$ & 4986 & 1148 & 2.5624 \\
\hline & $\mathrm{B}$ & 25267 & 6846 & 2.5518 \\
\hline & $\mathrm{C}$ & 62781 & 18164 & 2.535 \\
\hline & $\mathrm{D}$ & 89549 & 23673 & 2.5312 \\
\hline & $\mathrm{E}$ & 120083 & 32745 & 2.531 \\
\hline & $\mathrm{F}$ & 180356 & 52277 & 2.5308 \\
\hline \multirow{6}{*}{$\begin{array}{l}\text { Ratchet } \\
\text { Mechanism }\end{array}$} & $\bar{A}$ & 13187 & 4007 & 390.1 \\
\hline & $\mathrm{B}$ & 32113 & 7792 & 409.1 \\
\hline & $\mathrm{C}$ & 55574 & 12968 & 416.77 \\
\hline & $\mathrm{D}$ & 59988 & 15637 & 418.16 \\
\hline & $\mathrm{E}$ & 82921 & 19633 & 418.34 \\
\hline & $\mathrm{F}$ & 116639 & 28083 & 418.56 \\
\hline \multirow{6}{*}{$\begin{array}{l}\text { Release } \\
\text { Mechanism }\end{array}$} & $\mathrm{A}$ & 67111 & 38178 & 9.157 \\
\hline & $\mathrm{B}$ & 141680 & 84883 & 9.3707 \\
\hline & $\mathrm{C}$ & 177190 & 108033 & 11.016 \\
\hline & $\mathrm{D}$ & 191492 & 117792 & 12.127 \\
\hline & $\mathrm{E}$ & 223101 & 138168 & 12.141 \\
\hline & $\mathrm{F}$ & 291262 & 182953 & 12.143 \\
\hline
\end{tabular}

